\title{
THE MOMENTS OF THE AREA UNDER REFLECTED BROWNIAN BRIDGE CONDITIONAL ON ITS LOCAL TIME AT ZERO
}

\author{
FRANK B. KNIGHT \\ University of Illinois \\ Department of Mathematics, 1409 West Green Street \\ Urbana, IL 61801 USA
}

(Received May, 1999; Revised August, 1999)

This paper develops a recursion formula for the conditional moments of the area under the absolute value of Brownian bridge given the local time at 0 . The method of power series leads to a Hermite equation for the generating function of the coefficients which is solved in terms of the parabolic cylinder functions. By integrating out the local time variable, this leads to an integral expression for the joint moments of the areas under the positive and negative parts of the Brownian bridge.

Key words: Brownian Bridge, Local Time, Pitman Process, Method of M. Kac, Hermite Equations.

AMS subject classifications: $60 \mathrm{~J} 65,60 \mathrm{~J} 60$.

\section{Introduction}

\subsection{Review of the Methods and Results}

There is considerable literature on the integral functionals of Brownian motion, going back to M. Kac [5]. Recently, the results and methods have been unified by M. Perman and J.A. Wellner [9] who also give a good survey of the literature. The purpose of [9] was to obtain the law of the integral of the positive part of both Brownian motion and Brownian bridge. In short, they obtained the double Laplace transform of the laws of $A^{+}(t):=\int_{0}^{t} B^{+}(s) d s$ and $A_{0}^{+}:=\int_{0}^{1} U^{+}(t) d t$, where $B(s)$ and $U(t)$ are standard Brownian motion and Brownian bridge, respectively (Theorems 3.3 and 3.5 of [9]; actually they obtain the double Laplace transforms for an arbitrary linear combination of positive and negative parts). They also found (Corollary 5.1) a recursion formula for the moments. These results are obtained from excursion theory, by conditioning on the local time of $B$ at an independent exponential random instant, and appealing to previous known results of Kac, Shepp, etc.

Despite the considerable scope of these results, it seems to us worthwhile also to look at what can be done by conditioning on the local time $\ell_{0}$ of $U$ at $x=0$. In prin- 
ciple, all of the known results for the integrals of $U$ follow from the corresponding conditional law, by integrating over the (known) joint distribution of local time at zero and the positive sojourn. This is because $(a)$ the conditional law of the positive sojourn $S^{+}:=\int_{0}^{1} I(0, \infty)(U(t)) d t$ of $U$, given the local time at 0 , is known from $\mathrm{P}$. Lévy (see [7, Corollary 1] - that paper treats a problem analogous to the present but with the maximum replacing the area integral) and (b) given the positive sojourn $S^{+}$ and the local time $\ell_{0}$ at 0 , the local time processes of $U$ with parameters $x \geq 0$ and $x \leq 0$ are independent and distributed as the local time processes of reflected Brownian bridges with spans $S^{+}$and $1-S^{+}$, respectively (the corresponding assertions without conditioning on $\ell_{0}$ are false: given only $S^{+}$, the local time of $U$ at $x \geq 0$ is not equivalent in law to the local time of a reflected bridge of duration $S^{+}$, even if $x=0)$. Accordingly, we are led to look for the law of $\left(\int_{0}^{1}|U(t)| d t \mid \ell_{0}=x\right), 0 \leq x$. What we obtain below, however, is not an explicit expression for the law, but a recursion formula for the moments (as functions of $x$ ). The moments, in this case, determine the law and conversely, but experience in similar cases (for example, that of Brownian excursion; see L. Takács [14]) has shown that neither need follow easily from the other. Thus, finding the explicit conditional law seems to be still an open problem (as it is also for $A_{0}^{+}$, but to a lesser extent).

To describe our method, we consider the process defined by

$$
W(x):=\left(\ell(x), 1-\int_{0}^{x} \ell(u) d u\right), 0 \leq x
$$

conditional on $\ell(0)=\alpha \geq 0$, where $\ell(x)$ is the semimartingale (occupation) local time of $|U(t)|$ at $x \geq 0$. Thus, the second component is the residual lifetime of $|U(t)|$ above $x$ (we note the change of notation $-\ell(0):=2 \ell_{0}$ from above). Set $E:=$ $[0, \infty) \otimes(0,1]$. It is not hard to realize that $W(x)$ is a realization of a homogeneous Markov process on $E$, absorbed at $(0,0)$. This process, indeed, is the subject of a recent paper of J. Pitman [10] who characterizes it as the unique strong solution of a certain S.D.E., and it appears earlier in the paper of C. Leuridan [8], who obtained the form of the extended infinitesimal generator by an $h$-path argument. We propose to call this process the "Pitman process". Our requirements for this process are rather different from those of [10]. We wish to apply the method of Kac to the area functional

$$
\int_{0}^{\infty}\left(y-\int_{0}^{v} \ell(u) d u\right) d v=\int_{0}^{\infty} v \ell(v) d v \stackrel{d}{=} \int_{0}^{y}\left|U_{y}(u)\right| d u
$$

given $\ell(0)=\alpha$ and $\int_{0}^{\infty} \ell(u) d u=y$, where $U_{y}$ is a Brownian bridge of span $y \leq 1$. Thus, it is the integral of the second component of our process starting at $(\alpha, y)$. Consequently, we need to characterize this process $W$ via its infinitesimal generator, as a two dimensional diffusion whose semigroup has the Feller property. Much of this may be obvious to a very knowledgeable reader, but it provides orientation and it seems to us that the methods may be more widely of use. In any case, the reader who can accept Corollary 1.3.5 (with $A$ given by (1.2)) could go direction to Section 2 .

We need the results of [10, Proposition 3, Theorem 4] only to the extent that there exists a diffusion process $W=(X, Y)$ (a strong Markov process with continuous 
paths) on $E \cup(0,0)$ starting at $(x, y)$ and absorbed at the state $(0,0)$ at time $T_{0}:=$ $\inf \left\{\mathrm{t}>0: \int_{0}^{t} X(u) d u=y\right\}<\infty$, of which the process (1.0) is a realization with $x=$ $\alpha, y=1$, and the law of $X(\cdot)$ for this process is weakly continuous in its dependence on $(x, y)$. We also rely on the stochastic differential equation of [10] to determine the form of the generator of $W$, (see [8] for an alternative method).

Finally, we also need the scaling property $\left[10\right.$, Proposition 3 (iii)]. Let $P^{x, y}$ denote the law of $W$ starting at $(x, y) \in E$. Then the equality of law

holds.

$$
P^{x, y}\{X(\cdot) \in \cdot\}=P^{x / \sqrt{y}, 1}\{\sqrt{y} X(\cdot / \sqrt{y}) \in \cdot\}
$$

Our main assertion concerning $W$ is as follows.

Proposition 1.1.1: For $N>0, x \leq N$, let $W_{N}(t)$ denote $W\left(t \wedge T_{N}\right), 0 \leq t$, where $T_{N}:=\inf \{t: X(t)=N\}$, and let $E_{N}$ denote $[0, N] \otimes[0,1]$ with the segment $\{(x, 0)$, $0 \leq x \leq N\}$ identified to the single point $(0,0)$ and the quotient topology. Then $W_{N}$ has law that of a diffusion on the compact metrizable space $E_{N}$ absorbed at $\{x=N\} \cup\{y=0\}$, whose semigroup has the Feller property on $E_{N}$ and is strongly continuous at $t=0$, and with infinitesimal generator extending the operator

$$
A f(x, y):=\left(2 x \frac{\partial^{2}}{\partial x^{2}}+\left(4-\frac{x^{2}}{y}\right) \frac{\partial}{\partial x}-x \frac{\partial}{\partial y}\right) f(x, y) \text { for } f \in C_{c}^{2}\left(E_{N}^{0}\right)
$$

(interior compact support). The boundary segments $\{x=0,0<y \leq 1\}$ and $\{0 \leq x<N, y=1\}$ are inaccessible except at $t=0$.

Remark 1.1: It seems non-trivial to ascertain the behavior of $W$ starting at $(x, 1)$ as $x \rightarrow \infty$ (probably absorption at $(0,0)$ occurs instantly). Hence the need for $W_{N}$. One might hope to appeal to the fundamental uniqueness theorem of Stroock and Varadhan (as stated, for example, in Rogers and Williams [13]), but there are insuperable obstacles. To wit, the operator $A$ is not strictly elliptic, the coefficients are unbounded at $y=0$ and at $x=\infty$, and $A$ is undefined outside of $E$.

The proof of Proposition 1.1 .1 occupies Section 1.2 below. It uses a coupling argument, together with an extension of a strong comparison theorem of T. Yamada. It seems of interest that this last, originally stated only for diffusions on $R$, extends without any difficulty to the Pitman process on $R^{2}$ (Lemma 1.2.1). Knowing that we have Feller processes to work with, while not indispensable, makes for a neater treatment of Kac's method in Subsection 1.3. The form which we develop is doubtlessly familiar to many specialists, but we give a complete proof which should be adaptable to other analogous situations. In principle, the method applies to give $H_{\mu}(x):=$ $E^{x} \exp \left(-\mu \int_{0}^{T} V\left(X_{s}\right) d s\right)$ whenever $X$ is a Feller process absorbed on a boundary $\partial$ at time $T<\infty$, and $V(x)$ is sufficiently tractable. It then characterizes $H_{\mu}(x)$ as the unique bounded continuous solution of $(A-\mu V) H_{\mu}=0$ with $H_{\mu}=1$ on $\partial$, where $A$ denotes the generator of $X$. In other words, $H_{\mu}$ is harmonic for the process $X$ killed according to $\mu V$.

In Section 2, we specialize to the case when $V(x, y)=y$, and $X$ is the Pitman process absorbed on $\{x=N$ or $y=0\}$. We write $H_{\mu}=1+\sum_{n=1}^{\infty}(-\mu)^{n} a_{n}(x, y)$, and try an expansion $a_{n}=\sum_{0}^{\infty} b_{n, k}(x, y) x^{k}$. Then a scaling argument leads to $b_{n, k}=y^{3 n / 2}\left(x y^{-\frac{1}{2}}\right)^{k} c_{n, k}$, where $c_{n, k}$ are constants, and the problem reduces to determining $G_{n}(s):=\sum_{k=0}^{\infty} c_{n, k} s^{k}$. Some power series arguments lead (tentatively) to $G_{1}(s)=\frac{1}{2} \exp \left(\frac{s^{2}}{16}\right) D_{-1}\left(\frac{s}{2}\right)$, where $D_{-n}(s)$ denotes the parabolic cylinder function 
for $0 \leq n$. The key to the solution for $n>1$ lies in Lemma 2.3, where it emerges that $K_{n}(s):=s G_{n}(s)$ solves the inhomogeneous Hermite equation (2.13) (this remains a surprise to us). Since the forcing term $\left(-\frac{1}{2} G_{n-1}(s)\right)$ turns out inductively to be a finite linear combination of eigenfunctions $\left(G_{0} \equiv 1\right)$, this makes it possible to express the unique bounded solutions $G_{n}$ inductively in $n$, by a recursion formula for the coefficients (Theorem 2.4). This is our main result, but to establish it rigorously, by proving that the series for $H_{\mu}$ converges uniformly and absolutely on $E$ and satisfies the uniqueness conditions of Kac's method, occupies the rest of Section 2. Since the series is not summed explicitly, we do not find $H_{\mu}$ in an invertible form, but it yields the conditional moments, namely $n ! y^{3 n / 2} G_{n}\left(x y^{-\frac{1}{2}}\right), 1 \leq n$. The recursion formula (2.17) for the coefficients is not particularly simple, but no doubt it can be programmed on a computer if high-order moments are desired.

In Section 3, we derive closed form expressions for the moments of the areas of the absolute value and the positive part of a Brownian bridge in terms of the coefficients in Section 2. These are not as simple as previously known recursion (see [9]), but they are simpler (perhaps) given the coefficients of Section 2. Anyway, they provide more checks on Section 2, and the method leads in Theorem 3.6 to integrals for the joint moments of the areas of the positive and negative parts of Brownian bridge. These can be done explicitly in the simplest cases, but the general case (which hints at orthogonality relations among the parabolic cylinder functions) is beyond our capability.

\subsection{Proof of Proposition 1.1.1}

Let us show first that $T_{N} \wedge T_{0}$ tends to 0 uniformly in probability as $(x, y)$ tends to the absorbing boundary $\{x=N\} \cup\{y=0\}$ of $E_{N}$. There are really two separate problems here: one as $x$ increases to $N$ and the other as $y$ decreases to 0 . For $y>\delta>0$ as $x \rightarrow N$ the coefficients of $A$ near $\{x=N\}$ are bounded, in such a way that one can read off from the meaning of $A$ the uniform convergence in probability of $T_{N}$ to 0 . Unfortunately, to make this rigorous seems to require comparison methods as in Lemma 1.2.1 below (adapted from the one-dimensional case). Once the comparison is established, the convergence reduces to a triviality for one-dimensional diffusion with constant drift and need not concern us further.

The problem as $y \rightarrow 0$ is more interesting, and here it suffices to show that $T_{0}$ tends to 0 in probability as $y \rightarrow 0+$ uniformly in $x$ (for $W$, not for $W_{N}$ ). For $\epsilon>0$, let $E_{\epsilon}:=\left\{(x, y) \in E: x y^{-\frac{1}{2}} \leq \epsilon\right\}$, and let $R_{\epsilon}=\inf \left\{t>0: X_{t} \leq \epsilon Y_{t}^{\frac{1}{2}}\right\} \leq \infty$. Thus $R_{\epsilon}$ is the passage time to $E_{\epsilon}$, and it is a stopping time of $W$. We show first that $T_{0} \wedge R_{\epsilon}$ tends to 0 uniformly in $x$. Indeed, since $\left(\frac{d Y}{d t}\right) Y_{t}^{-\frac{1}{2}}=-X_{t} Y_{t}^{-\frac{1}{2}}<-\epsilon$ for $t<T_{0} \wedge R_{\epsilon}$, we have for the process starting at $(x, y) \in E-E_{\epsilon}, 2\left(y^{\frac{1}{2}}-Y^{\frac{1}{2}}\left(T_{0} \wedge R_{\epsilon}\right)\right)$ $>\epsilon\left(T_{0} \wedge R_{\epsilon}\right)$. Thus $T_{0} \wedge R_{\epsilon}<2 \epsilon^{-1} y^{\frac{1}{2}}$, uniformly in $x$ as asserted. Consequently, we see by the strong Markov property at time $T_{0} \wedge R_{\epsilon}$, that it suffices to show that $T_{0}$ is uniformly small in probability for $(x, y) \in E_{\epsilon} \cap\{y<\epsilon\}$ as $\epsilon \rightarrow 0+$.

To this effect, we use the scaling $(1.1)$ noting first that the process $Y(\cdot)$ may be 
included on the left if we include $y Y(\cdot / \sqrt{y})$ on the right. Indeed, $Y_{t}=y-\int_{0}^{t} X_{s} d s$, which for $P^{x / \sqrt{y}, 1}$ is equivalent to $y-\int_{0}^{t} \sqrt{y} X(s / \sqrt{y}) d s$, which equals $y(1-$ $\left.\int_{0}^{t / \sqrt{y}} X_{s} d s\right)=y Y(t / \sqrt{y})$ as asserted From this, it is seen that the $P^{x, y}$-law of $T_{0}$ equals the $P^{x / \sqrt{y}, 1}$-law of $y^{\frac{1}{2}} T_{0}$, and since $x y^{-\frac{1}{2}}<\epsilon$ and $y^{\frac{1}{2}}<\epsilon$ it is enough to show that $\lim _{N \rightarrow \infty} P^{x, 1}\left\{T_{0}>N\right\}=0$ uniformly for $x<\epsilon$ small. Here we can use the fact discussed in [10], that the $P^{0,1}$-law of $X_{t}$ is that of the local time of a standard Brownian excursion. As such, it does not return to the starting point 0 until 'time' $T_{0}$, i.e., $T_{0}$ is the excursion maximum value. Consequently, for small $\epsilon>0$, $P^{0,1}\left\{X_{t} Y_{t}^{-\frac{1}{2}}\right.$ reaches $\epsilon$ before $Y_{t}$ reaches $\left.\frac{1}{2}\right\}>\frac{1}{2}$. Then denoting the event in brackets by $S_{\epsilon}$, and setting $U(\epsilon)=\left\{\inf t>0: X_{t}=Y_{t}^{\frac{1}{2}} \epsilon\right\} \leq \infty$, we have by the strong Markov property

$$
\begin{gathered}
P^{0,1}\left\{T_{0}>N\right\} \geq E^{0,1}\left\{P^{X(U(\epsilon)), Y(U(\epsilon))}\left\{T_{0}>N\right\} ; S_{\epsilon}\right\} \\
=E^{0,1}\left(P^{(\epsilon, 1)}\left\{Y^{\frac{1}{2}}(U(\epsilon)) T_{0}>N\right\} ; S_{\epsilon}\right) \\
\geq P^{\epsilon, 1}\left\{T_{0}>N \sqrt{2}\right\} P\left(S_{\epsilon}\right) \geq \frac{1}{2} P^{\epsilon, 1}\left\{T_{0}>N \sqrt{2}\right\} .
\end{gathered}
$$

Since this uniform in $\epsilon$ (small), the assertion is now proved.

To derive the form (1.2) for the infinitesimal generator, it is enough to take $n=\infty$ and consider the semigroup of $W$ acting on the space $\mathscr{B}_{b}(E)$ of bounded, Borel functions. Then from $[10$, p. 1$]$, for $(x, y) \in E$ with $y>0$, the $P^{x, y}$-law of $W$ is that of the unique strong solution of

$$
d X_{u}=\left(4-X_{u}^{2} / Y_{u}\right) d u+2 \sqrt{X_{u}} d B_{u} ; d Y_{u}=-X_{u} d u ; \quad\left(X_{0}, Y_{0}\right)=(x, y),
$$

where the solution is unique up to the absorption time $T_{0}$ at $(0,0)$. Then by Itô's formula for $u<T_{0}$, we have $P^{x, y}$-a.s. for $f \in C_{c}^{2}\left(E_{N}^{0}\right)$,

$$
\begin{gathered}
f(W(u))=f(x, y)+\int_{0}^{u} f_{x}\left(W_{v}\right) 2 \sqrt{X_{v}} d B_{v} \\
+\int_{0}^{u}\left[2 f_{x x}\left(W_{v}\right) X_{v}+f_{x}\left(W_{v}\right)\left(4-X_{v}^{2} / Y_{v}\right)-f_{y}\left(W_{v}\right) X_{v}\right] d v .
\end{gathered}
$$

Since $f_{x}$ vanishes near $y=0$, we can take expectations to get

$$
\begin{gathered}
u^{-1}\left(E^{x, y} f\left(W_{u}\right)-f(x, y)\right) \\
=u^{-1} E^{x, y} \int_{0}^{u}\left(2 f_{x x}\left(W_{v}\right) X_{v}+f_{x}\left(W_{v}\right)\left(4-X_{v}^{2} / Y_{v}\right)-f_{y}\left(W_{v}\right) X_{v}\right) d v .
\end{gathered}
$$

Let $\epsilon=$ dist.(bdry $E$ to supp $f)>0$, where distance and boundary are Euclidean (without identifying the line $y=0)$, and let $\mathcal{C}=\left\{(x, y) \in E: \operatorname{dist}((x, y), \operatorname{supp} f) \leq \frac{\epsilon}{3}\right\}$. Starting at $(x, y) \in E-C$, the process must first reach $C$ before reaching supp $f$. 
Thus as $u \rightarrow 0+$ in (1.5) we get 0 uniformly for such $(x, y)$ provided that

$$
\lim _{u \rightarrow 0} u^{-1} P^{a, b}\left\{\sup _{s \leq u}\left|W_{s}-(a, b)\right|>\frac{\epsilon}{3}\right\}=0 \text { uniformly for }(a, b) \in \mathrm{C} .
$$

Similarly, for $(x, y) \in \mathcal{C},(1.5)$ tends uniformly to $A f(x, y)$ provided that (1.6) holds for every $\epsilon>0$. Thus the assertion (1.2) for the (strong) infinitesimal generator follows if we show that (1.6) holds for all $\epsilon>0$. Reducing $\epsilon$ if necessary, the coefficients of $A$ are uniformly bounded on an $\frac{\epsilon}{3}$ neighborhood $C_{\epsilon / 3}$ of $C$, and the "local character" assertion (1.6) is familiar for diffusion, at least in one-dimensional. Unfortunately, we lack a reference for dimension exceeding one, so for the sake of completeness we sketch a proof by reduction of $A$ to the one-dimensional case (fortunately $A$ is 'almost' one-dimensional). Indeed, the a.s. identity $Y(t)-b=-\int_{0}^{t} X(u) d u, t<$ $T_{0}$, shows that it suffices to prove (1.6) with $\left|X_{s}-a\right|$ in place of $\left|W_{s}-(a, b)\right|$. To this end, choose constants $0<c<d$ such that $c<4-x^{2} / y<d$ holds on $\mathrm{e}_{\epsilon / 3}$, and let $X_{t}^{(1)}$ (resp. $X_{t}^{(2)}$ ) be the solution of (1.3) starting at $a$ but with $c$ (resp. $d$ ) replacing the coefficient $4-x^{2} / y$ (using a single Brownian motion $B$ throughout).

Lemma 1.2.1: For $(a, b) \in \mathcal{C}$, we have $X_{t}^{(1)} \leq X_{t} \leq X_{t}^{(2)}, P^{(a, b)}$-a.s. for all $t \leq T:=T\left(E-e_{\epsilon / 3}\right)$ (the passage time of $W_{t}$ to $\left.E-e_{\epsilon / 3}\right)$.

Proof: The diffusion coefficient $\sigma(x)=2 \sqrt{x}$ is the same for all three processes, and satisfies $(\sigma(x)-\sigma(y))^{2} \leq 4|x-y|$, where $\int_{0+} x^{-1} d x=\infty$. Taking, for example, the semimartingale $\bar{X}_{t}^{(2)}-X_{t}$, suppose we have shown that its local time at 0 vanishes. Then we can repeat the proof of Yamada's comparison theorem from [11, IX (3.7)], using Tanaka's formula for continuous semimartingales [11, VI (1.2)], to get $E\left(X_{t \wedge T}-X_{t \wedge T}^{(2)}\right)^{+} \leq 0$, which suffices for the proof. The case of $X_{t}-X_{t}^{(1)}$ is analogous, so it remains to see that the local times vanish at 0 . The proof of $[11$, IX (3.4)] goes through without change in both cases, completing the argument.

Now to derive (1.6), note that because it is known that $P^{a, b}\left\{\sup _{s<u} \mid X_{s}^{(i)}-\right.$ $\left.a \mid>\frac{\epsilon}{6}\right\}=o(u), i=1$ or 2 , uniformly on $\mathcal{C}$, it follows from Lemma 1.2.1 that $P^{a, b}\left\{\sup _{s<u \wedge T}\left|X_{s}-a\right|>\frac{\epsilon}{6}\right\}=o(u)$ uniformly on $C$ as well. But since $\mid Y_{t}-$ $b \mid \leq t\left(a+\sup _{s}<t\left|X_{s}-a\right|\right)$, we have if $t<\epsilon(6 a+\epsilon)^{-1}$ and $\sup _{s} \leq t\left|X_{s}-a\right|<\frac{\epsilon}{6}$, also $\sup _{s}|| W_{s}-(a, b) \mid<\sqrt{2} \frac{\epsilon}{6}<\frac{\epsilon}{3}$, hence $t<T$. Thus conversely, for $u<$ $\epsilon(6 a+\epsilon)^{-千},\left\{\sup _{s \leq u \wedge T}\left|W_{s}-(a, b)\right|>\frac{\epsilon}{3}\right] \subset\left\{\sup _{s<u \wedge T}\left|X_{s}-a\right|>\frac{\epsilon}{6}\right\}$, and if $M$ is an upper bound of $a$ on $e$, then for $u<\epsilon(6 M+\epsilon)^{-1},\left\{\sup _{s} \leq u \wedge T \mid W_{s}-\right.$ $\left.(a, b) \mid>\frac{\epsilon}{3}\right\}$ has probability $o(u)$ uniformly on $C$. Since $P^{a, b}\left\{\sup _{s} \leq T \mid W_{s}-\right.$ $\left.(a, b) \mid\}>\frac{\epsilon}{3}\right\}=1$, this gives probability of $o(u)$ for $\left\{\sup _{s} \leq u \mid W_{s}-\right.$ $\left.(a, b) \mid>\frac{\epsilon}{3} ; u<T\right\} \cup\{u \geq T\}$, uniformly on $\mathrm{C}$, and this is not increased if we replace $\{u \geq T\}$ by $\left\{\sup _{s \leq u}\left|W_{s}-(a, b)\right|>\frac{\epsilon}{3} ; u \geq T\right\}$. Thus $P^{a, b}\left\{\sup _{s} \leq u \mid W_{s}-\right.$ $\left.(a, b) \mid>\frac{\epsilon}{3}\right\}=o(u)$, uniformly on $\mathrm{C}$ as required.

The last statement of Proposition 1.1.1 pertaining to $\{x=0\}$ is known for $P^{0, y}$ since then $X_{t}$ has the law of an excursion local time (see [10]). For $P^{x, y}$ it then follows from $P^{0, y}$ and the strong Markov property at the passage time to $x$. It remains to discuss the Feller property of the semigroup. Since $W_{t}$ was shown to be absorbed at $(0,0)$ for $P^{(x, y)}$, uniformly fast as $y \rightarrow 0+$, it is clear that we must identify the segment $\{(x, 0), 0 \leq x \leq N\}$ with $(0,0)$ in order to preserve continuity on the boundary of $E_{N}$. It is well-known and easy to check that the absorbed process $W_{N}$ is again a diffusion (on $\left.E_{N}\right)$. Let $T_{t}^{N}$ denote its semigroup on $\mathfrak{B}_{b}\left(E_{N}\right)$. Then by the above remarks, for $f \in \mathcal{C}\left(E_{N}\right)$ and $t>0, \lim _{(x, y) \rightarrow\left(x_{0}, y_{0}\right.} T_{t}^{N} f(x, y)=$ $f\left(x_{0}, y_{0}\right)$ uniformly for $\left(x_{0}, y_{0}\right) \in \operatorname{abs} E_{N}$, where $E_{N}$ is compact with $\operatorname{abs} E_{N}:=$ 
$\{(N, y), 0<y \leq 1\} \cup\{(0,0)\}$. Actually, the segment $\{0, x<N, y=1\}$ has yet to be discussed but it is obviously inaccessible except at $t=0$, and there is little difficulty now in seeing that $\lim _{t \rightarrow 0+} T_{t}^{N} f(x, y)=f(x, y)$ uniformly on $E_{N}$ for $f \in \mathcal{C}\left(E_{N}\right)$ since $\lim _{y \rightarrow 0+} f(x, y)=f(0,0)$ uniformly on $0 \leq x \leq N$ (here we can resort again to the comparison argument as in Lemma 1.2.1 to show that $\lim _{t \rightarrow 0+} P^{x, y}\left\{\mid X_{t}\right.$ $x \mid>\epsilon\}=0$ uniformly on $\left.E_{N}-\{y \leq \epsilon\}\right)$. In other words, we have strong continuity of $T_{t}^{N}$ at $t=0$, and it remains only to show that, for $f \in \mathcal{C}\left(E_{N}\right)$ and $t>0, T_{t}^{N} f$ is continuous on $E_{N}-\operatorname{abs} E_{N}$.

By the scaling property (1.1), we have

$$
T_{t}^{N} f(x, y)=E^{x, y} f\left(W_{N}(t)\right)=E^{x / \sqrt{y}, 1} f\left(\sqrt{y} X_{N / \sqrt{y}}(t / \sqrt{y}), y Y_{N / y}(t / \sqrt{y})\right) \text {. }
$$

By strong continuity of $T_{t}^{N}$, we have for $\epsilon>0$ and any $t_{1} \geq 0$, $\lim _{t_{2} \rightarrow t_{1}} P^{x, y}\left\{\left|W_{N}\left(t_{1}\right)-W_{N}\left(t_{2}\right)\right|>\epsilon\right\}=0$, uniformly in $(x, y)$ and $t_{1}$. Also, since absorption occurs uniformly fast near the absorbing boundary, it is seen that for $N_{1}<N_{2}<M$ fixed, $\lim _{N_{2}-N_{1} \rightarrow 0+} P^{x, 1}\left\{\left|W_{N_{2}}(t)-W_{N_{1}}(t)\right|>\epsilon\right\}=0$, uniformly in $t$ and $x \in E_{N_{1}}$ for a metric generating the topology of $E_{M}$.

Now let $\left(x_{n}, y_{n}\right) \rightarrow(x, y) \in E_{N}-\operatorname{abs} E_{N}$, and for each $n$ define two independent processes $W_{N}^{n}$ and $W_{N}$ on the same product probability space, where $W_{N}^{n}(0)=\left(x_{n}, y_{n}\right)$ and $W_{N}(0)=(x, y)$. Let

$$
T_{n}:=\inf \left\{t \geq 0: X_{N}^{n}(t)\left(Y_{N}^{n}(t)\right)^{-\frac{1}{2}}=X_{N}(t)\left(Y_{N}(t)\right)^{-\frac{1}{2}}\right\}
$$

where $\left(X_{N}^{n}, Y_{N}^{n}\right)=W_{N}^{n}$, etc. Since $0<Y_{N}(0)-Y_{N}(t)<N t$, it is seen (for example, using Lemma 1.2.1) that $\lim _{n \rightarrow \infty} T_{n}=0$ in law, and of course, each $T_{n}$ is a stopping time for the usual product filtration $\mathscr{F}_{n}(t)$. Then we have, if $|f|<c$,

$$
\begin{gathered}
\left|E^{x}{ }^{, y_{n}} f\left(W_{N}(t)\right)-E^{x, y} f\left(W_{N}(t)\right)\right| \leq 2 c P\left\{T_{n} \geq t\right\} \\
\left.+\mid E\left(E^{W_{N}^{n}\left(T_{n}\right)} f\left(t-T_{n}\right)-E^{W_{N}\left(T_{n}\right)} f\left(t-T_{n}\right)\right) ; T_{n}<t\right) \mid .
\end{gathered}
$$

As $n \rightarrow \infty$, the first term on the right tends to 0 . Setting

$$
Z^{n}\left(T_{n}\right):=X_{N}^{n}\left(T_{n}\right)\left(Y_{N}^{n}\left(T_{n}\right)\right)^{-\frac{1}{2}}=X_{N}\left(T_{n}\right)\left(Y_{N}\left(T_{n}\right)\right)^{-\frac{1}{2}}
$$

the difference in the second term becomes by (1.7)

$$
\begin{aligned}
& E^{Z^{n}\left(T_{n}\right), 1}\left[f \left(\sqrt{Y_{N}^{n}\left(T_{n}\right)} X_{N / \sqrt{Y_{N}^{n}\left(T_{n}\right)}}\left(t-T_{N}\right) / \sqrt{\left.Y_{N}^{n}\left(T_{n}\right)\right)}, Y_{N}^{n}\left(T_{n}\right)\right.\right. \\
& \cdot Y_{N / \sqrt{Y_{N}^{n}\left(T_{n}\right)}}\left(\left(t-T_{n}\right) / \sqrt{\left.Y_{N}^{n}\left(T_{n}\right)\right)}-f\right. \text { (analogous)] }
\end{aligned}
$$

where (analogous) has the scale factor $Y_{N}\left(T_{n}\right)$ in place of $Y_{N}^{n}\left(T_{n}\right)$. Now since $T_{n} \rightarrow 0$ in probability, it is clear that $Y_{N}^{n}\left(T_{n}\right) \rightarrow y$ and $Y_{N}\left(T_{n}\right) \rightarrow y$ in probability, and then by the remarks following (1.7), we see that the difference (1.9) tends to 0 in probability, viz. each term converges in probability to $E^{Z^{n}\left(T_{n}\right), 1} f\left(\sqrt{y} X_{N / \sqrt{y}}(t / \sqrt{y})\right.$, $\left.y Y_{N / \sqrt{y}}(t / \sqrt{y})\right)$ as $n \rightarrow \infty$, and since it is also bounded, (1.8) tends to 0 and the 
proof is complete.

Remark 1.2: It is possible, but tedious, to show that the law of a Feller diffusion on $E_{N}$ absorbed at $\{x=N\} \cup\{y=0\}$ and with strong generator satisfying (1.2) is thereby uniquely determined. For an indication of a proof, we observe that for $0<\epsilon$ small, the coefficients of $A$ satisfy a Lipschitz condition on $\widehat{E}_{N, \epsilon} ;=[\epsilon, N-\epsilon] \otimes[\epsilon, 1)$, in such a way that they may be extended from $\widehat{E}_{N, \epsilon_{\widehat{A}}}$ to $R^{2}$ and satisfy the conditions of $[13, \mathrm{~V}, 22]$. Thus if $(1.2)$ for the operator $\widehat{A}$ determined by the extended coefficients is assumed on $R^{2}$, there is a unique diffusion on $R^{2}$ which gives the unique solution to the "martingale problem." By optional stopping, this process absorbed on $\{x=\epsilon\} \cup\{x=N-\epsilon\} \cup\{y=\epsilon\}$ solves the martingale problem on $\widehat{E}_{N, \epsilon}$, and it is the unique such solution because any such can be extended to a solution on $R^{2}$ using the strong Markov property on the boundary. It remains only to let $\epsilon=\epsilon_{n} \rightarrow 0$, and to form the projective limit of these diffusions to obtain the law of $W_{N}$ uniquely.

\subsection{A Form of M. Kac's Method for Functionals of an Absorbed Process}

We turn now to establishing a variant of Kac's method for obtaining the law of functionals of $W_{N}$. For this we need to introduce a "killing" of $W_{N}$ according to the desired functional. But as an introduction to the problem we first make some observa-

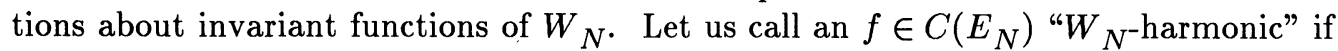
$f\left(W_{N}(t)\right)$ is a $P^{x, y}$-martingale for $(x, y) \in E_{N}$. We claim that $f$ is $W_{N}$-harmonic if $f \in C\left(E_{N}\right) \cap C^{2}\left(E_{N}^{0}\right)$ and $A f=0$ on $E_{N}^{0}$ (the interior of $E_{N}$ ). Suppose first that $f \in C_{c}^{2}\left(E_{N}^{0}\right)$ (compact support). Then $f$ is in the domain of the strong generator and by Dynkin's Formula we have $E^{x, y} f\left(W_{N}(t)\right)=f(x, y)+E^{x, y} \int_{0}^{t} A f\left(W_{N}(s)\right) d s=$ $f(x, y)$. Thus the martingale property follows by the Markov property of $W_{N}$. Now supposing only $f \in \mathrm{C}^{2}\left(E_{N}^{0}\right)$, set $E_{N, \epsilon}=[\epsilon, N-\epsilon] \otimes[\epsilon, 1-\epsilon]$ and note that by standard smoothing argument there is an $f_{\epsilon} \in \mathrm{e}_{c}^{2}\left(E_{N}^{0}\right)$ with $f_{\epsilon} \equiv f$ on $E_{N, \epsilon}$. It follows by optional stopping that, for $(x, y) \in E_{N, \epsilon}, f\left(W_{N}\left(t \wedge T_{\epsilon}\right)\right)$ is a $P^{x, y^{*}}$-martingale where

$$
T_{\epsilon}:=\inf \left\{t \geq 0: W_{N}(t) \in\{x=\epsilon \text { or } N-\epsilon\} \cup\{y=\epsilon\}\right\} .
$$

Now $f$ is uniformly bounded and, by continuity of paths we have $\lim _{\epsilon \rightarrow 0} T_{\epsilon}=T_{\text {abs }}$ : = $\inf \left\{t>0: W_{N}(t) \in \operatorname{abs} E_{N}\right\}$ for $(x, y) \in E_{N}^{0}$. It follows that for $(x, y) \in E_{N}^{0}$, $f\left(W_{N}\left(t \wedge T_{\text {abs }}\right)\right)$ is a $P^{x, y}$-martingale. Since $W_{N}(t)=W_{N}\left(t \wedge T_{\text {abs }}\right)$, and the result is trivial for $(x, y) \in \operatorname{abs} E_{N}$, this finishes the argument except for $P^{x, 1}$. But, of course, the Markov property for $P^{x, 1}$ shows that $f\left(W_{N}(t+\epsilon)\right)$ is a martingale given $\left\{W_{N}(s), s \leq \epsilon\right\}$ for every $\epsilon>0$, and along with right-continuity of $W_{N}$ at $t=0$ this suffices trivially.

Remark 1.3.1: The converse assertion that if $f$ is $W_{N}$-harmonic then $A f \equiv 0$ on $E_{N}^{0}$ is probably valid, but it is not needed for the purposes here. For applications it is the solutions of $A f \equiv 0$ which give the "answers." We note also the expression

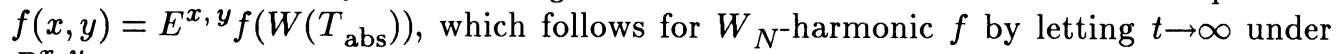
$P^{x, y}$.

Now fix a $V(x, y) \in C^{+}\left(E_{N}\right)$, and let $E_{N, \Delta}=E_{N} \cup\{\Delta\}$, where $\Delta$ is adjoined to $E_{N}$ as an isolated point. For each $P^{x, y},(x, y) \in E_{N}$, let $e$ be an independent exponential random variable adjoined to the probability space of $W_{N}$, and introduce: 
Definition 1.3.2: The process $W_{N}$ killed according to $V$ is

$$
W_{N, V}(t)=\left\{\begin{array}{cc}
W_{N}(t) ; & t<T(\Delta) \\
\Delta ; & t \geq T(\Delta)
\end{array}\right.
$$

where $T(\Delta)=\inf \left\{t: \int_{0}^{t \wedge} T_{\text {abs } V}\left(W_{N}(s)\right) d s \geq e\right\}$, with the inclusion of an extra path $\left(W_{N, V}(t)=\Delta, \forall t \geq 0\right):=w_{\Delta}$, and $P^{\Delta}\left\{w_{\Delta}\right\}=1$.

Noting carefully that there is no "killing" (passage to $\Delta$ ) on abs $E_{N}$, so that $T(\Delta)=\infty$ for $P^{x, y}$ if $(x, y) \in \operatorname{abs} E_{N}$, we have:

Theorem 1.3.3: With the initial probabilities $P^{x, y}$ from $W_{N},(x, y) \in E_{N}$, $W_{N, V}(t)$ becomes a Feller process on $E_{N, \Delta}$, strongly continuous at $t=0$, with continuous paths except for (possibly) a single jump from $E_{N}$ to $\Delta$. The (strong) infinitesimal generator is given by $A_{V} f:=A(f)-V f$ for $f \in \mathrm{C}_{c}^{2}\left(E_{N, \Delta}^{0}\right),(x, y) \in E_{N}$, and $A_{V} f(\Delta)=0$. The process is absorbed on abs $E_{N} \cup \Delta$.

Proof: (Sketch) The killing formula used here goes back to G.A. Hunt, and is well-known to yield a strong Markov process from the Feller process $W_{N}$. In proving the strong continuity and the Feller property, the main thing to use is that the killing occurs uniformly slowly on $E_{N}$, i.e. $\lim _{\epsilon \rightarrow 0} P^{x, y}\{T(\Delta)<\epsilon\}=0$ uniformly on $E_{N}$. This not only suffices to derive the strong continuity at $t=0$ from that of $W_{N}$, but it also preserves the main point of the coupling argument used to prove the Feller property, namely, that the coupling time $T_{n}(\leq \infty)$ tends to 0 in law when in its definition $W_{N}$ is replaced by $W_{N, V}$. But a difficulty arises with the analog of (1.9) since, for general $V, W_{N, V}$ does not obey the scaling property (1.7). Instead, we have to introduce the killing operation on the paths $\left(\sqrt{Y_{N}^{n}\left(T_{n}\right)} X_{N / \sqrt{Y_{N}^{n}\left(T_{n}\right)}}\right.$ $\left(\left(t-T_{n}\right) / \sqrt{\left.Y_{N}^{n}\left(T_{n}\right)\right)}\right.$, etc.) in (1.9) starting at $\left(X_{N}^{n}\left(T_{n}\right), Y_{N}^{n}\left(T_{n}\right)\right)$, and analogously without superscript $n$. But there will be no change in the result if we use the same process $W$ starting at $\left(Z^{n}\left(T_{n}\right), 1\right)$ in both terms of the difference. In other words, we base the two futures after $T_{n}$ on a single process $W(t)$ (using the probability kernel $Z^{n}\left(T_{n}\right) \rightarrow P^{Z^{n}\left(T_{n}\right), 1}$ to define the conditional law of the future at $T_{n}$ given $\mathcal{F}_{n}\left(T_{n}\right)$, in the usual way for Markov processes). This implies that in introducing the killing operations into the two terms we use the path with scale factor $\left(Y_{N}^{n}\left(T_{n}\right)\right)^{\frac{1}{2}}$ for the first, and $\left(Y_{N}\left(T_{n}\right)\right)^{\frac{1}{2}}$ for the second, but the same path $W(t), W(0)=\left(Z^{n}\left(T_{n}\right), 1\right)$, for each. Then convergence in probability of the scale factors to $y$ implies that the

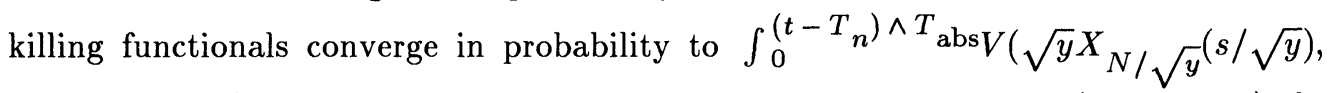
$\left.y Y_{N / \sqrt{y}}(s / \sqrt{y})\right) d s$, i.e. their difference converges to 0 . If we use (as we may) the same exponential random variable $e$ to do the killing for both, it is clear that, with conditional probability near 1 , either both are killed by time $t-T_{n}$ or neither, in such a way that the Feller property holds for the semigroup $T_{t}^{N, V}$ of ${ }_{W}{ }_{N, V}$.

Turning to the assertion about the infinitesimal generator of $T_{t}^{N, V}$, note first that $f(\Delta)=0$ for $f \in \mathrm{C}_{c}^{2}\left(E_{N, V}^{0}\right)$. We have

$$
t^{-1}\left(T_{t}^{N, V} f-f\right)=t^{-1}\left(T_{t}^{N} f-f\right)-t^{-1} E^{\bullet}\left(f\left(W_{N}(t)\right) ; W_{N, V}(t)=\Delta\right)
$$

on $E_{N}$, while the same expression is 0 at $\Delta$. The first term on the right converges to $A(f)$ uniformly on $E_{N}$. Using (1.6) as before, we may assume $\left|W_{N}(t)-(x, y)\right|<\frac{\epsilon}{3}$, 
with error $o(t)$ uniformly on $C$ as $t \rightarrow 0$. Then $f\left(W_{N}(t)\right)$ may be replaced by $f(x, y)$ for small $t$, uniformly on $E_{N}$, and we are left with $f(x, y) t^{-1}{ }_{P}^{(x, y)}$ $\left(\int_{0}^{t \wedge} \operatorname{abs} V\left(W_{N}(s)\right) d s>e\right)$. This vanishes outside $\operatorname{supp} f$, and $P^{(x, y)}\left(T_{\text {abs }}<t\right)=$ $o(t)$ uniformly on $\operatorname{supp} f$. Thus we can extend the integral to $t$, and then

$$
\begin{gathered}
P^{(x, y}\left(\int_{0}^{t} V\left(W_{N}(s)\right) d s>e\right)=1-E^{x, y} \exp \left(-\int_{0}^{t} V\left(W_{N}(s)\right) d s\right) \\
=E^{x, y}\left(\int_{0}^{t} V\left(W_{N}(s)\right) d s\right)+o(t) \\
=t V(x, y)+o(t),
\end{gathered}
$$

uniformly on $E_{N}$. This completes the proof, the last assertion being obvious.

We come now to the key method (of Kac).

Theorem 1.3.4: Continuing the potation of Theorem 1.3.3, for $\mu \geq 0$ and $(x, y) \in$ $E_{N}$ set $H_{\mu}(x, y)=E^{x, y} \exp \left(-\mu \int_{0}^{T}\right.$ abs $\left.V\left(W_{N}(s)\right) d s\right)$. Suppose there exists an $H \in$ $\mathrm{e}\left(E_{N}\right) \cap C^{2}\left(E_{N}^{0}\right)$ with $A(H)-\mu V H=0$ on $E_{N}^{0}$ and $H=1$ on abs $E_{N}$. Then $H=$ $H_{\mu}$ on $E_{N}$.

Proof: We have $\left.H_{\mu}(x, y)=P^{(x, y}\right)\left\{W_{N, \mu V}\right.$ reaches abs $E_{N}$ before time $\left.T(\Delta)\right\}=$ $P^{(x, y)}\{T(\Delta)=\infty\}$, when $T(\Delta)$ is defined for $\mu V$ in place of $V$. Clearly, $H_{\mu}=1$ on $\operatorname{abs} E_{N}$, and if we set $H_{\mu}(\Delta)=0$ then $H_{\mu}$ is harmonic for the process $W_{N, \mu V}$, at least if it is continuous. Indeed we have $H_{\mu}(x, y)=E^{x, y} H_{\mu}\left(W_{N, \mu V}\left(T_{a b s} \wedge T(\Delta)\right)\right)$, and it follows by the Markov property of $W_{N, \mu V}$ that $H_{\mu}\left(W_{N, \mu V}(t)\right)$ is a $P^{x, y_{-}}$ martingale for $(x, y) \in E_{N}$.

On the other hand, if we set $H(\Delta)=0$, then $H=H_{\mu}$ on $\operatorname{abs} E_{N} \cup \Delta$, and we claim that $H$ (being continuous by assumption ) is harmonic for $W_{N, \mu V}$. As in the discussion above for $W_{N}$, this is taken to mean that $H\left(W_{N, \mu V}(t)\right)$ is a $P^{x, y}$-martingale, $(x, y) \in E_{N}$. The proof is much the same as above for $W_{N}$ (see (1.10)) only now the martingale has a (possible) jump. In short, using $E_{N, \epsilon}=[\epsilon, N-\epsilon] \otimes[\epsilon, 1-\epsilon]$ as before but $H$ in place of $f$ with $H_{\epsilon}=H$ on $E_{N, \epsilon}, H_{\epsilon} \in C_{c}^{2}\left(E_{N}^{0}\right)$, and $H_{\epsilon}(\Delta)=0$, optional stopping of the martingale $H_{\epsilon}\left(W_{N, V}(t)\right)$ for $(x, y) \in E_{N, \epsilon}$ shows that

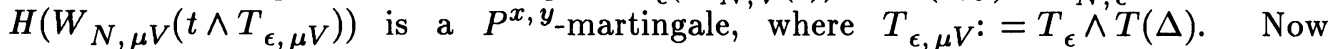

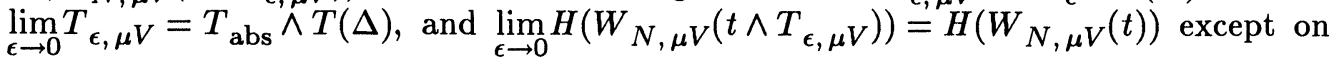
the $P^{x, y}$-nullset where $T_{\text {abs }}=T(\Delta)$. By dominated convergence of conditional expectations, $H\left(W_{N, \mu V}(t)\right)$ is $P^{x, y}$-martingale if $(x, y) \in E_{N}^{0}$. The assertion is trivial for $(x, y) \in \operatorname{abs} E_{N} \cup \Delta$, and letting $t \rightarrow \infty$ we have $H(x, y)=E^{x, y} H\left(W_{N, \mu V}\right.$ $\left.\left(T_{\text {abs }} \wedge T(\Delta)\right)\right)$. This is the same as with $H_{\mu}$ in place of $H$, so the proof is complete (except if $y=1$, but that case now follows from the continuity of $H$, which implies that $H_{\mu}$ extends by continuity to $y=1$ ).

What we need for Section 2 below is a form of Theorem 1.3.3 applying to the case $N=\infty$, or rather, to the limit as $N \rightarrow \infty$. This means replacing $W_{N}$ by the process $W$ of (1.0) $f f$. We first modify the definition of $E$ slightly, by identifying the line $\{(0, x), 0 \leq x<\infty\}$ with $(0,0)$, so that $E_{N} \subset E$ with the relative topology. We do not compactify $E$; however, we know from [10] that on $E P^{(x, y)}\left\{T_{0}<\infty\right\}=1$, where $T_{0}$ is the passage time to $(0,0)$ as before. Moreover, since $4-\frac{x^{2}}{y} \leq 4$ on $E$, it 
follows as in the comparison Lemma 1.2.1 that $P^{x, y}\left\{X_{t} \leq X_{t}^{(2)}, t \leq T_{0}\right\}=1$ for $(x, y) \in E$, where $X_{t}^{(2)}$ is a diffusion with generator $2 x \frac{d^{2}}{d x^{2}}+4 \frac{d}{d x}$ on $R^{+}$. For $X_{t}^{(2)}$ there are no "explosions" ( $\infty$ is inaccessible) $([6,4.5])$ and it follows by comparison that as $N \rightarrow \infty, P^{x, y}\left\{X_{t}\right.$ reaches $N$ before $\left.T_{0}\right\}$ tends to 0 uniformly on compact sets of $E$. It follows easily that the semigroup $T_{t}$ of $W$ preserves $C_{b}(E)$ (but of course, it is not strongly continuous at $t=0$ ), and its infinitesimal generator has the form $A f$ for $f \in \mathrm{C}_{c}^{2}\left(E^{0}\right), A$ as in (1.2), just as for $T_{t}^{N}$.

For $V \in \mathrm{C}_{b}^{+}(E)$ (bounded, continuous on $E$ ) we define $W_{V}$ from $W$ just as in Definition 1.3.2 for $W_{N, V}$, where $T_{\text {abs }}$ is replaced by $T_{0}$. The scaling (1.7) remains valid for $T_{t}$ (only it is a little simpler here without absorption at $N$ ), and the coupling argument remains valid to show that the semigroup $T_{t}^{V}$ of $W_{V}$ preserves $C_{b}(E)$. Likewise, the argument after (1.9) for the generator of $T_{t}^{N, V}$ goes through for $T_{t}^{V}$. Thus we see that Theorem 1.3 .3 carries over to $W_{V}$ with only the changes noted: the generator is $A_{V} f$ for $f \in \mathrm{C}_{c}^{2}\left(E_{\Delta}^{0}\right)$ and the process is absorbed on $(0,0) \cup \Delta$. We also have the analog of Theorem 1.3.4 as follows:

Corollary 1.3.5: Set $H_{\mu}(x, y)=E^{x, y} \exp \left(-\mu \int_{0}^{T_{0}} V(W(s)) d s\right),(x, y) \in E$, with $V \in \mathrm{C}_{b}^{+}(E), \quad \mu \geq 0$. Suppose there exists an $H \in \mathrm{C}_{b}(E) \cap \mathrm{e}^{2}\left(E^{0}\right)$ with $A(H)-$ $\mu V H=0$ on $E^{0}$ and $H(0,0)=1$. Then $H_{\mu}=H$ on $E$.

Proof: There is nothing really new here, but it recapitulates the former proof. We have $H_{\mu}(x, y)=P^{x, y}\left\{T_{0}<T(\Delta)\right\}$ where $T(\Delta)$ corresponds to $\mu V$, and so $H_{\mu}(x, y)$ is $W_{\mu V}$-harmonic (if we set $H_{\mu}(\Delta)=0$ ) apart from continuity considerations. On the other hand, $H$ is continuous by assumption (and we set $H(\Delta)=0)$. Since $H=H_{\mu}$ on $(0,0) \cup \Delta$ and $H_{\mu}(x, y)=E^{x, y} H_{\mu}\left(W_{\mu V}\left(T_{0} \wedge T(\Delta)\right)\right)$, it suffices to show that $H(x, y)=E^{x, y} H\left(W_{\mu V}\left(T_{0} \wedge T(\Delta)\right)\right),(x, y) \in E$. We note from the definition that if we absorb $W_{\mu V}$ on $\{x=N\}$, for $(x, y) \in E_{N}$ we get a

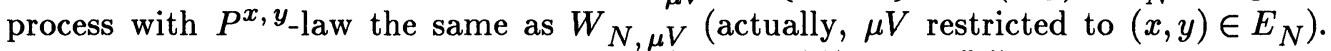
The proof of Theorem 1.3.4 shows that $H\left(W_{N, \mu V}(t)\right)$ is a $P^{x, y}$-martingale. Now for $(x, y) \in E, W_{N, \mu V}(\cdot)$ and $W_{\mu V}(\cdot)$ coincide for $N$ sufficiently large, (depending on the path), so it follows by bounded convergence of conditional expectations that $H\left(W_{\mu V}(t)\right)$ is a martingale for $P^{x, y}$. Then letting $t \rightarrow \infty$ we obtain the assertion.

\section{Derivation of the Conditional Moments}

We now specialize Corollary 1.3 .5 to $V(x, y) \equiv y$, writing for brevity $H_{\mu}$ for $H_{\mu y}$. Then, as noted following (1.0), we have $H_{\mu}(x, y)=E^{x, y}\left(\exp \left(-\mu \int_{0}^{y}\left|U_{y}(s)\right|\right.\right.$ - ds $\left.) \mid \ell_{y}(0)=x\right)$ where $U_{y}$ is a Brownian bridge of term $y$, and $\ell_{y}(v)$ the local time at $v$ of $\left|U_{y}\right|$. Thus $H_{\mu}(x, y)$ is the Laplace transform whose inversion gives the law of $\left(\int_{0}^{y}\left|U_{y}(s)\right| d s \mid \ell_{y}(0)=x\right)=\left(\int_{0}^{\infty} v \ell_{y}(v) d v \mid \ell_{y}(0)=x\right)$. We do not solve $(A-$ $\mu y) H_{\mu}=0$ per se, but instead we assume an expansion $H_{\mu}(x, y)=\sum_{n=0}^{\infty} \frac{(-\mu)^{n}}{n !} E^{x, y}$ $\cdot\left(\int_{0}^{T_{0}} Y(v) d v\right)^{n}$, and then solve recursively for the terms. It is shown that the series converges for $\mu \in R$ and satisfies the conditions for $H_{\mu}$. Hence the expansion is justified and the conditional moments are

$$
E^{x, y}\left(\int_{0}^{T_{0}} Y(v) d v\right)^{n}=E\left(\left(\int_{0}^{y}\left|U_{y}(s)\right| d s\right)^{n} \mid \ell_{y}(0)=x\right)
$$


and they determine the (conditional) law of $\left(\int_{0}^{y}\left|U_{y}(s)\right| d s \mid \ell_{y}(0)=x\right)$.

In order not to prejudice the notation, let us write formally

$$
H_{\mu}(x, y)=1+\sum_{n=1}^{\infty}(-\mu)^{n} a_{n}(x, y)
$$

and try to solve for the functions $a_{n}(x, y)$. First we note from the scaling property (1.1) that we have

$$
H_{\mu}(x, y)=H_{\mu c}{ }^{3}\left(x c^{-1}, y c^{-2}\right), 0<c .
$$

Indeed, since $Y_{v}=\int_{v}^{\infty} X(u) d u$, we have for $B^{2} \in \mathscr{B}_{E}$,

$$
P^{x, y}\left\{\left(X_{v}, Y_{v}\right) \in B^{2}\right\}=P^{x / c, y / c^{2}}\left\{\left(c X_{\frac{v}{c}}, c^{2} Y_{\frac{v}{c}}\right) \in B^{2}\right\}
$$

and so $\int_{0}^{T_{0}} Y_{v} d v\left(=\int_{0}^{\infty} Y_{v} d v\right)$ for $P^{x, y}$ is equivalent to $c^{3} \int_{0}^{\infty} Y_{v} d v$ for $P^{x / c, y / c^{2}}$, as asserted. Then we may impose

$$
a_{n}(x, y)=c^{2 n} a_{n}\left(x c^{-1}, y c^{-2}\right), c>0,1 \leq n
$$

Remark: We do not need to justify (2.2) rigorously, because it leads to the explicit solution, which is unique and verifiable.

Recalling (Corollary 1.3.5) that the equation satisfied by $H_{\mu}$ is

$$
A H_{\mu}=\left(2 x \frac{d^{2}}{d x^{2}}+\left(4-\frac{x^{2}}{y}\right) \frac{d}{d x}-x \frac{d}{d y}\right) H_{\mu}=\mu y H_{\mu} \text { on } E
$$

we have by matching powers of $\mu$,

$$
A a_{1}=-y ; A a_{n+1}=-y a_{n}, 1 \leq n,
$$

and we are lead to guess the existence of solutions in the form

$$
\begin{gathered}
a_{0}(x, y)=1 \\
a_{n}(x, y)=\sum_{k=0}^{\infty} b_{n, k}(y) x^{k} ; \quad 1 \leq n
\end{gathered}
$$

(by formal analogy of $A$ with the heat operator $\frac{d^{2}}{d x^{2}}-\frac{d}{d t}$, when $y$ replaces $t$ ).

We remark that $a_{n}(0, y)\left(=b_{n, 0}(y)\right)$ should be the $n^{\text {th }}$ moment over $n !$ of the integral of the Brownian excursion of length $y$. By scaling this is $c_{n} y^{\frac{3 n}{2}}$ where $c_{n}:=\frac{M_{n}}{n !}$ with $M_{n}$ denoting the $n^{\text {th }}$ moment for the integral of standard Brownian excursion. These moments figure prominently in Takács [13], where $M_{k}, k \leq 10$, are tabulated (Table 4) and a recursion formula is given. Here they provide a check on our answers. When we work out the $b_{n, k}(y)$ by power series method, it turns out that the series of even and odd terms commence with $b_{n, 0}(y)=c_{n} y^{\frac{3}{2} n}$, and $b_{n, 1}(y)=$ $-\frac{c_{n}-1}{4} y^{n}\left(\right.$ where $c_{0}=1$ ), respectively, where at first the $c_{n}$ are arbitrary constants whose identity is known only from the (assumed) excursion connection. However later on, when we sum the series in terms of parabolic cylinder functions $D_{-n}$, it emerges that the values of $c_{n}$ are dictated uniquely by the behavior (limit 0 ) of the solution as $y \rightarrow 0$. Thus it turns out that the case of the excursion $(x=0)$ follows as a 
consequence.

When we substitute the series (2.5) into (2.4), it emerges that there is a solution in the form $b_{n, k}(y)=c_{n, k} y^{\frac{(3 n-k)}{2}}$, so that $c_{n, 0}=c_{n}$ and $c_{n, 1}=-\frac{c_{n-1}}{4}$. Indeed, granted a solution of the form (2.5), this form for $b_{n, k}$ follows by the scaling (2.2). Thus we expect

$$
a_{n}(x, y)=y^{\frac{3 n}{2}} \sum_{k=0}^{\infty} c_{n, k}\left(x y^{\left.-\frac{1}{2}\right)^{k}}\right.
$$

and the summation of the series reduces to identifying the generating functions

$$
G_{n}(s):=\sum_{k=0}^{\infty} c_{n, k} s^{k} ; \quad G_{0}(s)=1
$$

Let us go through the case $n=1$ directly (although it is a consequence later of more general considerations) since (unlike $n=2$ ) we can derive the result by direct summation of the series, and it shows where the functions $D_{-n}$ come from in this problem.

Lemma 2.1: There exists a bounded continuous solution $a_{1}(x, y)$ on $E$ of $A a_{1}=$ $-y$ having the form (2.6)-(2.7) with $c_{1,0}=c_{1}=M_{1}$ and $G_{1}(s)=\frac{1}{2} \exp \left(\frac{s^{2}}{16}\right) D_{-1}\left(\frac{s}{2}\right)$, where $D_{-1}$ is the parabolic cylinder function $[15,16.6]$. Moreover, $\lim _{-1} a_{1}(x, y)=0$ and this is the unique such solution.

Proof: Substituting (2.5) for $a_{1}$ into (2.4) gives $b_{1,1}=\frac{-y}{4}$ from the constant term (in $x$ ), and then $b_{1,0}^{\prime}=12 b_{1,2}$ from the coefficients of $x$. From the scaling (2.2) we need $c^{3} b_{1,0}\left(y c^{-2}\right)=b_{1,0}(y)$, hence $b_{1,0}(y)=\widehat{c} y^{\frac{3}{2}}$ for a constant $\widehat{c}$ not yet determined. Then $b_{1,2}=\frac{\widehat{c}}{8} y^{\frac{1}{2}}$, and continuing in this way, each $b_{1,2 k+1}$ follows from $b_{1,2 k-1}$ uniquely, as does $\left.b_{1,2(k+1}\right)$ from $b_{2 k}$ (the latter all have the factor $\widehat{c}$ ). Thus we get formally

$$
\begin{gathered}
a_{1}(x, y)=\left(-\sum_{k=0}^{\infty} 4^{-(k+1)}((2 k+1)(2 k-1) \cdots 1)^{-1} y^{-k+1} x^{2 k+1}\right)+\widehat{c} y^{\frac{3}{2}} \exp \left(\frac{x^{2}}{8 y}\right) \\
=\frac{-x y}{4} \sum_{k=0}^{\infty}\left(\frac{x^{2}}{8 y}\right)^{k}\left(\frac{1}{\frac{3}{2} \cdot \frac{5}{2} \cdots\left(k+\frac{1}{2}\right)}\right)+\widehat{c} y^{\frac{3}{2}} \exp \left(\frac{x^{2}}{8 y}\right)
\end{gathered}
$$

where for $k=0$ the expression $\frac{3}{2} \cdot \frac{5}{2} \cdots\left(k+\frac{1}{2}\right):=1$. Now comparison with the series expansion of the confluent hypergeometric function $M_{k, m}([15, \mathrm{XVI}, 16.1])$ shows that this becomes

$$
a_{1}(x, y)=\left(\frac{-x y}{4} M_{-\frac{1}{4}, \frac{1}{4}}\left(\frac{x^{2}}{8 y}\right)\left(\frac{x^{2}}{8 y}\right)^{\frac{-3}{4}} \exp \frac{x^{2}}{16 y}\right)+\widehat{c} y^{\frac{3}{2}} \exp \left(\frac{x^{2}}{8 y}\right)
$$

Similarly, by Kummer's first formula [ibid, 16.11] we get

$$
\begin{gathered}
\widehat{c} y^{\frac{3}{2}} \exp \frac{x^{2}}{8 y}=\widehat{c} y^{\frac{3}{2}} \exp \frac{x^{2}}{16 y} M_{\frac{1}{4},-\frac{1}{4}}\left(\frac{-x^{2}}{8 y}\right)\left(\frac{-x^{2}}{8 y}\right)^{-\frac{1}{4}} \\
=\widehat{c}\left(\exp \frac{x^{2}}{16 y}\right) y^{\frac{7}{4}} 8^{\frac{1}{4}} x^{\frac{-1}{2}} M_{\frac{-1}{4}, \frac{-1}{4}}\left(\frac{x^{2}}{8 y}\right)
\end{gathered}
$$


and hence

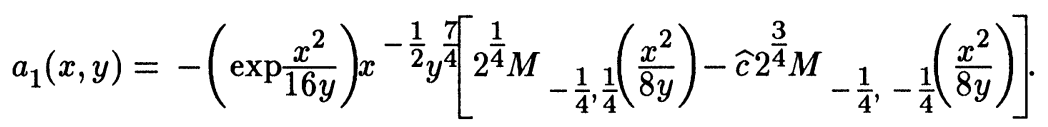

Here the functions $M$ on the right are unbounded as $\frac{x^{2}}{8 y} \rightarrow \infty$, but the expression of the Whittaker function $W_{k, m}$ in terms of $M_{k, \pm m}$ from [ibid, 16.41] shows that if $\widehat{c}=\frac{\sqrt{2 \pi}}{4}$, then $(2.10)$ becomes

$$
a_{1}(x, y)=2^{-\frac{3}{4}}\left(\exp \frac{x^{2}}{16 y}\right) x^{-\frac{1}{2} y^{\frac{7}{4}} W}-\frac{1}{4},-\frac{1}{4}\left(\frac{x^{2}}{8 y}\right)
$$

The asymptotic expansion of $W$ [ibid, 16.3] gives $W_{-\frac{1}{4},-\frac{1}{4}}(x) \sim \exp \left(-\frac{x}{2}\right) x^{-\frac{1}{4}}$ as $x \rightarrow \infty$, so that $a_{1}(x, y) \sim \frac{y^{2}}{x}$ which tends to 0 as $\frac{x^{2}}{y} \rightarrow \infty$ (if $x<k$ fixed then $\frac{x}{y} \rightarrow \infty$, and $\frac{y^{2}}{x}<\frac{y}{x} \rightarrow 0$, but if $x \rightarrow \infty$ then $\left.\frac{y^{2}}{x}<\frac{1}{x} \rightarrow 0\right)$. On the other hand, if $\frac{x}{y} \rightarrow \infty$, while $\frac{x^{2}}{y}<k$ fixed, then $\left(\frac{y}{x}\right)^{\frac{1}{2}} y^{\frac{3}{2}} \rightarrow 0$ and so (2.11) tends to 0 as asserted. As for boundedness, we have limit 0 if $x \rightarrow 0$ with $\frac{x^{2}}{y}>\epsilon>0$ since $x^{-\frac{1}{2}} y^{\frac{7}{4}}=\left(\frac{y}{x^{2}}\right)^{\frac{1}{4}} y^{\frac{3}{2}}$ and $y \rightarrow 0$, so it only

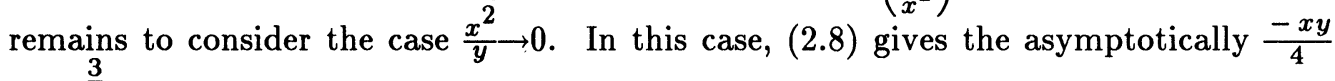
$+\widehat{c} y^{\frac{3}{2}}$, where $y<1$ and $x \rightarrow 0$, so boundedness follows.

As for the uniqueness assertion, let $\widehat{a}_{1}$ denote the difference of two such solutions. Then $A \widehat{a}_{1}=0$, and it follows as in Section 1 that $\widehat{a}_{1}(W(t))$ is a bounded martingale for $P^{x, y},(x, y) \in E$. Since $Y(t)=\int_{t}^{T_{0}}(X(s)) d s$, we see that as $t \uparrow T_{0}$ (and $\left.X(t) \rightarrow 0\right)$ we have $Y(t)<\left(T_{0}-t\right) X(t)$ when $X(t)=\max _{s \geq t} X(s)$, hence for a random sequence $t_{n} \rightarrow T_{0}$. Then $X\left(t_{n}\right) / Y\left(t_{n}\right) \rightarrow \infty$, and so $\widehat{a}_{1}\left(W\left(t_{n}\right)\right) \rightarrow 0$. Hence $\widehat{a}_{1}(W(t)) \rightarrow 0$ as $t \rightarrow T_{0}$, and it follows that $\widehat{a}_{1}(x, y) \equiv 0$ as asserted.

It remains to express (2.11) in terms of $D_{-1}$. By definition of $G_{1}$, and using the definition of $D_{-1}$ in [ibid, 16.5], we have as asserted

$$
\begin{aligned}
& G_{1}(s)=2^{-\frac{3}{4}} \exp \left(\frac{s^{2}}{16}\right) s^{-\frac{1}{2}} W_{\frac{1}{4},-\frac{1}{4}}\left(\frac{s^{2}}{8}\right) \\
& =2^{-\frac{3}{4}} 2^{\frac{1}{4}}\left(\frac{s}{2}\right)^{\frac{1}{2}} s^{-\frac{1}{2}} \exp \left(\frac{s^{2}}{16}\right) D_{-1}\left(\frac{s}{2}\right) \\
& =\frac{1}{2} \exp \left(\frac{s^{2}}{16}\right) D_{-1}\left(\frac{s}{2}\right) \text {. }
\end{aligned}
$$

Remark 2.1: From the expression of $W$ in terms of $M$ ([ibid, 16.41]) it follows that $W_{-\frac{1}{4},-\frac{1}{4}}(x)=W_{\frac{1}{4}, \frac{1}{4}}(x)$ for $x \geq 0$. Then by [ibid, 16.2] we get $G_{1}(s)=$ $2^{-\frac{1}{2}} \exp \left(\frac{s^{2}}{8}\right) \operatorname{erfc}\left(\frac{s}{\sqrt{8}}\right), 0 \leq s$. This is useful below in Section 3. Using the recurrence formula for $D_{n}$, and $D_{0}(s)=\exp \left(-\frac{s^{2}}{4}\right)$, an analogous "erfc representation" of $G_{n}$ holds for every $n$, but seems too complicated to be very useful.

Turning to the case of general $n$, two separate substitutions into (2.4), one for even 
powers of $x$ and one for odd, reveal that both sequences $c_{n, 2 k}$ and $c_{n, 2 k+1}$ satisfy the same recursion relation for $k>0$. Namely, we have (setting $c_{0,0}=1 ; c_{0, k}=0$, $1 \leq k)$,

Lemma 2.2: For any initial sequence $c_{n}:=c_{n, 0}, 1 \leq n$, the form (2.6) and (2.7) for the solution of (2.4) implies that

$c_{n, 1}=\frac{-c_{n-1}}{4}, 1 \leq n ;$ and $c_{n, k}=\frac{(3 n+k-2) c_{n, k-2}-2 c_{n-1, k-1}}{4 k(k+1)}, 2 \leq k$.

This determines the $c_{n, k}$ uniquely.

In view of the extra $k$ in the denominator of $c_{n, k}$, we can see by induction on $n$ that the series $G_{n}(s)$ converges absolutely for all real $s$. But we will find $G_{j}(s)$ bounded for $s \geq 0$ if and only if $c_{j}=\frac{M_{j}}{j !}, j \leq n$, and then $n ! a_{n}(x, y)=E^{x, y}\left(\int_{0}^{T_{0}}\right.$ $\cdot Y(v) d v)^{n}$. It also can be seen from (2.12) that whenever $c_{j}>0$ for all $j$, we have $c_{n, 2 k}>0, c_{n, 2 k+1}<0$ for all $n, k$.

Taking generating functions of both sides of (2.12) leads to:

Lemma 2.3: Let $K_{n}(s):=s G_{n}(s), 0 \leq n$. Then (for any choice of $c_{n}$ )

$$
K_{n}^{\prime \prime}(s)-\frac{s}{4} K_{n}^{\prime}(s)-\left(\frac{3 n-1}{4}\right) K_{n}(s)=-\frac{1}{2} G_{n-1}(s), 1 \leq n .
$$

Proof: By (2.12) we have

$$
\begin{gathered}
G_{n}(s)=c_{n}-\frac{1}{4} c_{n-1} s+\frac{1}{4} s \sum_{j=0}^{\infty} \frac{3 n-3+(j+3)}{(j+2)(j+3)} c_{n, j} s^{j+1} \\
-\frac{s}{2} \sum_{j=1}^{\infty} \frac{c_{n-1, j} s^{j}}{(j+1)(j+2)}=c_{n}-\frac{1}{4} c_{n-1} s \\
+\frac{3}{4}(n-1) s^{-1} \sum_{k=2}^{\infty} \frac{c_{n, k-2} s^{k+1}}{k(k+1)}+\frac{1}{4} \sum_{k=2}^{\infty} \frac{c_{n, k-2} s^{k}}{k} \\
-\frac{1}{2} s^{-1} \sum_{k=2}^{\infty} \frac{c_{n-1, k-1}}{k(k+1)} s^{k+1}=c_{n}-\frac{1}{4} c_{n-1} s \\
+\frac{3}{4}(n-1) s^{-1} \int_{0}^{s} \int_{0}^{v} u G_{n}(u) d u d v+\frac{1}{4} \int_{0}^{s} v G_{n}(v) d v \\
-\frac{1}{2} s^{-1} \int_{0}^{s} \int_{0}^{v}\left(G_{n-1}(u)-c_{n-1}\right) d u d v .
\end{gathered}
$$

Multiplying through by $s$, and differentiating twice yields the assertion.

Remark: It is seen that, in fact, (2.12) is equivalent to (2.13) with the initial conditions $K_{n}(0)=0, K_{n}^{\prime}(0)=c_{n}, 1 \leq n$.

The eigenvalue problem $X^{\prime \prime}-x X^{\prime}-\lambda X=0$ is known [2, Darling and Siegert] to have the solutions $\left(\exp \frac{x^{2}}{4}\right) D_{-\lambda}(x)$ and $\left(\exp \frac{x^{2}}{4}\right) D_{-\lambda}(-x)$. For $\lambda \geq 0$, the only solutions bounded for $x \geq 0$ are $c\left(\exp \frac{x^{2}}{4}\right) D_{-\lambda}(x)$. (For a discussion of the eigenfunc- 
tions of the Hermite equations, see [1]).

By change of variables of the homogeneous form of (2.13), namely

$$
X^{\prime \prime}(s)-\frac{s}{4} X^{\prime}(s)=\left(\frac{3 n-1}{4}\right) X(s)
$$

has $c\left(\exp \frac{s^{2}}{16}\right) D_{-(3 n-1)}\left(\frac{s}{2}\right)$ as unique solution bounded for $s \geq 0$. To give a probabilistic interpretation, let $V(t), 0 \leq t$ be an Ornstein-Uhlenbeck velocity process on $R$ with generator $\Omega F=F^{\prime \prime}-\frac{x}{4} F^{\prime}$, and for initial value $x>0$ let $T_{0}=\inf \{t>0: V(t)=$ $0\}$. Then $E^{x} e^{-\left(\frac{3 n-1}{4}\right) T_{0}}=D_{-(3 n-1)}^{-1}(0) \cdot\left(\exp \frac{x^{2}}{16}\right) D_{-(3 n-1)}\left(\frac{x}{2}\right)$. This representation of the Laplace transform of a diffusion passage time distribution is familiar ([4, $4.10])$. Let us moreover observe at this point, that by the recurrence formula for $D_{n}$ $[15,16.61]$ we have

$$
D_{-n}(0)=\sqrt{\pi} 2^{-\frac{n}{2}} \Gamma^{-1}\left(\frac{n+1}{2}\right), 0 \leq n
$$

so $\left(\exp \frac{x^{2}}{16}\right) D_{-(3 n-1)}\left(\frac{x}{2}\right)$ is real-valued and decreases to 0 as $x \rightarrow \infty$.

Our general method now consists in solving recursively equation (2.13), dividing by $s$ after each step to obtain the new $G_{n}$. One method would be to apply the resolvent operator of the Ornstein-Uhlenbeck process, but that is unnecessary because, as it turns out, each $G_{n}$ is a finite linear combination of eigenfunctions. Let us rederive the expression for $G_{1}$ of Lemma 2.1 to illustrate the method. Since $G_{0}=1$ we want to solve $K_{1}^{\prime \prime}-\frac{s}{4} K_{1}^{\prime}-\frac{1}{2} K_{1}=-\frac{1}{2}$. A particular solution is $K \equiv 1$ (an eigenfunction, incidentally). The only solutions of the homogeneous equation bounded for $s \geq 0$ are $c\left(\exp _{16} \frac{s^{2}}{16}\right) D_{-2}\left(\frac{s}{2}\right)$. The general solution bounded for $s \geq 0$ is thus $1+$ $c\left(\exp _{16}^{s^{2}}\right) D_{-2}\left(\frac{s}{2}\right)$, and we must choose $c$ in such a way that division by $s$ at 0 gives a finite $G_{1}$. Clearly there is exactly one $c$ possible. By the recurrence formula [loc. sit] $D_{-2}(z)=-z D_{-1}(z)+D_{0}(z)$ where $D_{0}(0)=1$, so we have only to take $c=-1$ and the solution becomes $G_{1}(s)=\frac{1}{2}\left(\exp _{\frac{s^{2}}{16}}\right) D_{-1}\left(\frac{s}{2}\right)$, in accordance with Lemma 2.1 .

The general case is quite analogous, only complicated. The following is the main result of our paper.

Theorem 2.4: For $n \geq 1$ and $G_{n}$ as in (2.7) (see also (2.6), (2.0)) we have

$$
G_{n}(s)=\left(\exp \frac{s^{2}}{16}\right) \sum_{j=n}^{3 n-2} d_{j, n} D_{j}\left(\frac{s}{2}\right)
$$

where the coefficients $d_{j, n}$ are determined by $d_{j, n}=0$ if $j-n$ is odd, and otherwise

$$
\begin{aligned}
& d_{1,1}=\frac{1}{2} \\
& d_{n+1+2 i, n+1}= \\
& 2^{\frac{n}{2}+i} \Gamma\left(\frac{n+1}{2}+i\right) \sum_{j=n}^{N(i)} \frac{2^{-\frac{j}{2}}}{\Gamma\left(\frac{j+1}{2}\right)} \frac{d_{j, n}}{3 n+2-j} ; N(i) \stackrel{\text { def }}{=} n+2(i \wedge(n-1)) \\
& \quad=2^{+i-1} \Gamma\left(\frac{+1}{2}+i\right) \sum_{j=0}^{i \wedge(n-1)} \frac{2-j}{\Gamma\left(\frac{n+1}{2}+j\right)} \frac{d_{n+2 j, n}}{(n+1-j)} ; 0 \leq i \leq n .
\end{aligned}
$$


Proof: We proceed by induction on $n$. For $n=1$ the assertion has been shown. Suppose that (2.16) holds for $n(\geq 1)$, and let us derive (2.17) for $d_{j, n+1}$. We first require a particular solution $X_{p}$ of

$$
X_{p}^{\prime \prime}-\frac{s}{4} X_{p}^{\prime}-\frac{3 n+2}{4} X_{p}=-\frac{1}{2}\left(\exp \frac{s^{2}}{16}\right) \sum_{j=n}^{3 n-2} d_{j, n} D_{-j}\left(\frac{s}{2}\right)
$$

in view of Lemma 2.3, where $d_{n+1, n}=d_{n+3, n}=\cdots=d_{3(n-1), n}=0$. But by (2.14) we have, setting $X=\left(\exp \frac{s^{2}}{16}\right) D_{-j}\left(\frac{s}{2}\right)$, the equation $X^{\prime \prime}-\frac{s}{4} X^{\prime}-\frac{j}{4} X=0$, so we can take

$$
X_{p}=-\frac{1}{2}\left(\exp \frac{s^{2}}{16}\right) \sum_{j=n}^{2 n-2} 4 \frac{d_{j, n}}{j-(3 n+2)} D_{-j}\left(\frac{s}{2}\right) .
$$

The general solution of $(2.18)$ bounded for $s>0$ has the form $c\left(\exp _{\frac{s^{2}}{16}}\right) D_{-(3 n+2)^{\left(\frac{s}{2}\right)}}$ $+X_{p}(s)$, so it remains to choose $c$ such that this times $s^{-1}$ remains bounded at $s=$ 0 (clearly $c$ is uniquely determined), and to simplify the division by $s$. To this end, we will need the following simple lemma.

Lemma 2.5: For $2 \leq 2 i \leq j$,

$$
\begin{aligned}
& D_{-j}(z)=(j-1)^{-1}(j-3)^{-1} \ldots(j-2 i+1)^{-1} D_{-j+2 i}(z) \\
& -z\left[(j-1)^{-1} D_{-(j-1)}(z)+((j-1)(j-3))^{-1} D_{-(j-3)}(z)+\ldots\right. \\
& \left.+((j-1)(j-3) \ldots(j-2 i+1))^{-1} D_{-j+2 i-1}(z)\right] \\
& =2^{-i} \Gamma\left(\frac{j+1}{2}-1\right) \Gamma^{-1}\left(\frac{j+1}{2}\right) D_{-j+2 i}(z) \\
& -z \Gamma^{-1}\left(\frac{j+1}{2}\right)\left[2^{-1} \Gamma\left(\frac{j-1}{2}\right) D_{-(j-1)}(z)+2^{-2} \Gamma\left(\frac{j-3}{2}\right) D_{-(j-3)}(z)\right. \\
& \left.+\ldots+2^{-i} \Gamma\left(\frac{j+1}{2}-i\right) D_{-j+2 i-1}(z)\right] \text {. }
\end{aligned}
$$

Proof: The first equality follows by repeated application of the recursion formula $D_{n+1}(z)-z D_{n}(z)+n D_{n-1}(z)=0$, starting with $n-1=-j([15$, loc. sit., 16.61]), and the second then follows by repeated use of $\Gamma(n+1)=n \Gamma(n)$.

To determine first the constant $c$, we only need to use (2.15). It follows that

$$
c=2^{\frac{3}{2} n} \Gamma\left(\frac{3(n+1)}{2}\right) \sum_{j=n}^{3 n-2} \frac{4 d_{j, n^{2}}{ }^{-\frac{j}{2}}}{(j-(3 n+2)) \Gamma\left(\frac{j+1}{2}\right)}
$$

and hence

$$
\begin{aligned}
& \left(\exp -\frac{s^{2}}{16}\right) G_{n+1}(s)=s^{-1} \\
& \cdot \sum_{j=n}^{3 n-2}\left(\sqrt{2}^{3 n-j} \Gamma\left(\frac{3(n+1)}{2}\right) \Gamma^{-1}\left(\frac{j+1}{2}\right) 4 D_{-(3 n+2)}\left(\frac{s}{2}\right)-2 D_{-j}\left(\frac{s}{2}\right)\right) \frac{d_{j, n}}{j-(3 n+2)}
\end{aligned}
$$


Now in the special case $n=1=j$, we apply Lemma 2.5 with $j=5$ to get $D_{-5}(z)=8^{-1} D_{-1}(z)-z\left[4^{-1} D_{-4}(z)+8^{-1} D_{-2}(z)\right]$, and substituting into the above gives $d_{2,2}=8^{-1}, d_{2,4}=4^{-1}$, as in $(2.1 \overline{7})$. In other cases $n>1$, we apply Lemma 2.5 twice, once to $D_{-}(3 n+2)$ with $j=3 n+2$ and $2 i=2(n+1)$, and once to $D_{-j}$ with $j=j$ and $2 i=j-n+2$. (Note that when $j-n+2$ is odd, $d_{j, n}=0$ by induction hypothesis.) Then the $s^{-1}$ cancels with $z=\frac{s}{2}$ and we are left with

$$
\begin{gathered}
\left(\exp -\frac{s^{2}}{16}\right) G_{n+1}(s)=-\frac{1}{2} \sum_{j=n}^{n-2} \Gamma^{-1}\left(\frac{j+1}{2}\right)\left[\left\{\sqrt{2}^{3 n-j+2} \Gamma\left(\frac{3 n+1}{2}\right) D_{-(3 n+1)}\left(\frac{s}{2}\right)\right.\right. \\
\left.+\sqrt{2}^{3 n-j} \Gamma\left(\frac{3 n-1}{2}\right) D_{-(3 n-1)}\left(\frac{s}{2}\right)+\ldots+\sqrt{2}^{n-j} \Gamma\left(\frac{n+1}{2}\right) D_{-(n+1)}\left(\frac{s}{2}\right)\right\} \\
-\left\{\Gamma\left(\frac{j-1}{2}\right) D_{-(j-1)}\left(\frac{s}{2}\right)+2^{-1} \Gamma\left(\frac{j-3}{2}\right) D_{-(j-3)}\left(\frac{s}{2}\right)+\ldots\right. \\
\left.\left.\quad+\sqrt{2}^{n-j} \Gamma\left(\frac{n+1}{2}\right) D_{-(n+1)}\left(\frac{s}{2}\right)\right\}\right] \frac{d_{j, n}}{j-(3 n+2)} \\
=-\frac{1}{2} \sum_{j=n}^{3 n-1} \Gamma\left(\frac{j+1}{2}\right)\left[\sqrt{2}^{3 n-j+2} \Gamma\left(\frac{3 n+1}{2}\right) D_{-(3 n+1)}\left(\frac{s}{2}\right)\right. \\
\left.+\sqrt{2}^{3 n-j} \Gamma\left(\frac{3 n-1}{2}\right) D_{-(3 n-1)}\left(\frac{s}{2}\right)+\ldots+2 \Gamma\left(\frac{j+1}{2}\right) D_{-(j+1)}\left(\frac{s}{2}\right)\right] \frac{d_{j, n}}{j-(3 n+2)}
\end{gathered}
$$

We see that terms in $D_{-(3 n+1)}$ and $D_{-(3 n-1)}$ occur for all $j=n+2 i, 0 \leq i \leq$ $n-1$, while terms in $D_{-(3 n-3)}$ occur for $0 \leq i \leq n-2, \ldots$ terms in $D_{-}(n+1)$ occur only for $i=0(j=n)$, and there are no terms in $D_{-k}$ for $n+1+k$ odd. Collecting coefficients, we get

\begin{tabular}{|c|c|c|c|c|c|c|c|c|c|c|c|}
\hline$k=$ & 1 & 2 & 3 & 4 & 5 & 6 & 7 & 8 & 9 & 10 & $\ldots$ \\
\hline$n=1$ & $\frac{1}{2}$ & 0 & 0 & 0 & 0 & 0 & 0 & 0 & 0 & 0 & $\ldots$ \\
\hline 2 & 0 & $\frac{1}{8}$ & 0 & $\frac{1}{4}$ & 0 & 0 & 0 & 0 & 0 & 0 & $\ldots$ \\
\hline 3 & 0 & 0 & $\frac{1}{48}$ & 0 & $\frac{1}{8}$ & 0 & $\frac{5}{8}$ & 0 & 0 & 0 & $\ldots$ \\
\hline 4 & 0 & 0 & 0 & $\frac{2^{-7}}{3}$ & 0 & $2^{-5}$ & 0 & $11 \cdot 2^{-5}$ & 0 & $\frac{11}{4}$ & 0 \\
\hline
\end{tabular}

$$
d_{n+1+2 i, n+1}=-2^{\frac{n}{2}+i} \Gamma\left(\frac{n+1}{2}+i\right) \sum_{j=n}^{N(i)} \frac{2^{-\frac{j}{2}} d_{j, n}}{\Gamma\left(\frac{j+1}{2}\right)(j-(3 n+2))} ; 0 \leq i \leq n .
$$

This reduces immediately to the expression of (2.17) as required.

Remark 2.2: It is clear that $d_{k, n} \geq 0$ for all $(k, n)$. By simple calculations we get for $d_{k, n}, 1 \leq n \leq 4$, the values

Table 2.1. Values of $d_{k, n}$

Substitutions of $(2.15)$ into $(2.16)$ gives values of $G_{n}(0)\left(=\frac{M_{n}}{n !}\right)$ equal to those of 
[14].

As a further check on the formula (2.17) let us rederive the equation $G_{n+1}^{\prime}(0)=$ $-\frac{1}{4} G_{n}(0), 1 \leq n$, which is clear from (2.12) of Lemma 2.2. Using the formula $D_{n}^{\prime}(z)=n D_{n-1}(z)-\frac{1}{2} z D_{n}(z)$ from $[15,16.61]$ gives $D_{-n}^{\prime}(0)=-n D_{-n-1}(0)=$ $-n 2^{-\frac{n+1}{2}} \sqrt{\pi} \Gamma^{-1}\left(\frac{n+2}{2}\right)$, and substitution into (2.16) and (2.17) yields (somewhat tediously)

$$
\begin{aligned}
G_{n+1}^{\prime}(0)= & \frac{-\sqrt{\pi}}{2}\left(\sum_{j=n}^{3 n-2} \frac{2^{-\frac{j}{2}} d_{n, j}}{\Gamma\left(\frac{j+1}{2}\right)(3 n-2+j)} \cdot\left(\frac{3 n-2+j}{2}\right)\right) \\
& =-\frac{1}{4} \sum_{j=n}^{3 n-2} d_{j, n} D_{-j}(0), \text { as required. }
\end{aligned}
$$

We have obtained the functions $a_{n}(x, y)=y^{\frac{3 n}{2}} G_{n}\left(x y^{-\frac{1}{2}}\right)$ for $(2.0)$ which, along with $a_{0}(x, y)=1$, gives our solution for $H_{\mu}(x, y)$. But to establish it rigorously we still have to justify summation of the series. Adding (2.4), for each $n$ we have $A\left(\sum_{j=0}^{n+1}(-\mu)^{j} a_{j}(\cdot)\right)=\mu y\left(\sum_{j=0}^{n}(-\mu)^{j} a_{j}(\cdot)\right)$. We will show that as $n \rightarrow \infty$, the series converges uniformly in $(x, y) \in E$, in any finite interval of $\mu$ and that term-byterm differentiation of power series twice is justified (it suffices to justify differentiation of $\sum_{n=1}^{\infty} \mu^{n} G_{n}(s)$ twice in $s$ ) to obtain $A H_{\mu}=+\mu y H_{\mu}$, i.e. (2.3) as required. Indeed, such $H_{\mu}$ satisfies the boundary condition $H_{\mu}(x, o)=1$. Note that the limit 1 is even uniform in $x$ as soon as $\sum_{n=1}^{\infty}(-\mu)^{n} G_{n}(s)$ is bounded for $s \geq 0$, because of the powers $y^{\frac{3 n}{2}}, 1 \leq n$, in $a_{n}(x, y)$. Moreover, it is not hard to see that $0 \leq G_{n}(s) \downarrow 0$ as $s \rightarrow \infty$ for each $n \geq 1$ : we have remarked above that $d_{k, n}$ are nonnegative for all $k, n$ while by the representation as transform of Ornstein-Uhlenbeck passage probabilities to zero (see $(2.14) \mathrm{ff}$.) it follows that $0 \leq\left(\exp \frac{s^{2}}{16}\right) D_{-j}\left(\frac{s}{2}\right) \downarrow 0$ as $s \rightarrow \infty$ for $0 \leq j$.

Remark: Since $G_{n}(s)$ has its maximum on $R^{+}$at $s=0$, where $G_{n}(0)$ is $(n !)^{-1}$ times the $n^{\text {th }}$ moment $M_{n}$ of the integral of the Brownian excursion, it would seem that convergence of the series could easily be proved by using the recursion formula of Takács [14] for the excursion moments. But this argument is circular - we do not know that $G_{n}(0)$ has the required interpretation until our solution is rigorously established.

Lemma 2.6: The series $\sum_{n=1}^{\infty}(-\mu)^{n} G_{n}(s)$ converges uniformly and absolutely in $s \geq 0$ for every $\mu \in R$ and may be differentiated (twice) term-by-term for $s>0$.

Proof: For convergence, by the preceding remarks it suffices to show that $\sum_{n=1}^{\infty} \mu^{n} G_{n}(0)<\infty$. Let us set $e_{n+1+2 i, n+1}:=d_{j, n} D_{-j}(0)=d_{j, n} \sqrt{\pi}^{-\frac{j}{2}} \Gamma^{-1}$ $\cdot\left(\frac{j+1}{2}\right)$, from $(2.15)$. Then $(2.17)$ becomes

$$
e_{n+1+2 i, n+1}=2^{\frac{n}{2}+i-1-\frac{n+1}{2}}-\frac{i \Gamma\left(\frac{n+1}{2}+i\right)}{\Gamma\left(\frac{n+2}{2}+i\right)} \sum_{j=0}^{i \wedge(n-1)} \frac{e_{n+2 j, n}}{(n+1-j)}
$$




$$
=2^{-\frac{3}{2} \Gamma\left(\frac{n+1}{2}+i\right)} \frac{i \wedge}{\Gamma\left(\frac{n+2}{2}+i\right)} \sum_{j=0}^{(n-1)} \frac{e_{n+2 j, n}}{n+1-j}
$$

and of course, $G_{n}(0)=\sum_{i=0}^{n-1} e_{n+2 i}$.

Now as $k \rightarrow \infty, \frac{\Gamma(k)}{\Gamma\left(k+\frac{1}{2}\right)}=\frac{\Gamma^{2}(k) 2^{2 k-1}}{\sqrt{\pi} \Gamma(2 k)}=\frac{((k-1) !)^{2} 2^{2 k-1}}{(2 k-1)(2 k-2) ! \sqrt{\pi}} \sim \frac{1}{\sqrt{k}}$, where we used the $\Gamma$-duplication formula and [3, VII (2.6)]. So we get, as $n \rightarrow \infty$,

$$
\begin{aligned}
& G_{n+1}(0) \cong 2^{-\frac{3}{2}} \sum_{i=0}^{n} \frac{1}{\sqrt{\frac{n+2}{2}+i}} \sum_{j=0}^{i \wedge(n-1)} \frac{e_{n+2 j, n}}{n+1-j} \\
& <2^{-\frac{3}{2}} \sum_{i=0}^{n} \frac{1}{\sqrt{\frac{n+2}{2}+i}} \frac{1}{n+1-i}\left(\sum_{j=0}^{i \wedge(n-1)} e_{n+2 j, n}\right) \\
& =2^{-\frac{3}{2}}\left[\frac{1}{\sqrt{\frac{n+2}{2}+n}} \sum_{j=0}^{n-1} e_{n+2 j, n}+\sum_{j=0}^{n-1} e_{n+2 j, n} \sum_{i=j-1}^{n} \frac{1}{\sqrt{\frac{n+2}{2}+i}}\left(\frac{1}{n+1-i}\right)\right] \\
& <2^{-\frac{3}{2}}\left(\frac{1}{\sqrt{n}}\right) G_{n}(0)+\sum_{j=0}^{n-1} e_{n+2 j, n}\left(\frac{1}{\sqrt{\frac{n}{2}}}\left(\frac{1}{1}+\frac{1}{2}+\ldots+\frac{1}{n+1}\right)\right) \\
& \sim \frac{1}{2} G_{n}(0)\left(\frac{1}{\sqrt{n}} \ln n\right) .
\end{aligned}
$$

By the ratio test, we see that the series converges as required. As to the differentiation with respect to $s$, we have (as in Remark 2.2)

$$
\begin{gathered}
\frac{d}{d s}\left(\left(\exp \frac{s^{2}}{16}\right) D_{-n}\left(\frac{s}{2}\right)\right)=\left[\left(\frac{s}{8}-\frac{s}{8}\right) D_{-n}\left(\frac{s}{2}\right)-\frac{n}{2} D_{-n-1}\left(\frac{s}{2}\right)\right]\left(\exp \frac{s^{2}}{16}\right)<0, \\
\frac{d^{2}}{d s^{2}}\left(\left(\exp \frac{s^{2}}{16}\right) D_{-n}\left(\frac{s}{2}\right)\right)=\left[\frac{n(n+1)}{4} D_{-n-2}\left(\frac{s}{2}\right)+\frac{s n}{16} D_{-n-1}\left(\frac{s}{2}\right)-\frac{s n}{16} D_{-n-1}\left(\frac{s}{2}\right)\right] \\
\cdot\left(\exp \frac{s^{2}}{16}\right)=\frac{n(n+1)}{4} D_{-n-2}\left(\frac{s}{2}\right)>0, \text { and similarly } \\
\frac{d^{3}}{d s^{3}}\left(\exp \frac{s^{2}}{16}\right) D_{-n}\left(\frac{s}{2}\right)=-\frac{n(n+1)(n+2)}{8} D_{-n-3}\left(\frac{s}{2}\right)\left(\exp \frac{s^{2}}{16}\right)<0 .
\end{gathered}
$$

Thus we see that the (two) derivatives are bounded in absolute value by their values at 0 , which are at most $\frac{n(n+1)}{4} D_{-n}(0)$. This does not change the radius of conver gence of the power series. Since the series of derivatives converges uniformly in $s \geq 0$, we have 


$$
\begin{gathered}
\frac{d}{d s} \sum_{n=1}^{\infty} \mu^{n} G_{n}\left(\frac{s}{2}\right)=\frac{d}{d s} \sum_{n=1}^{\infty} \mu^{n}\left(G_{n}(0)+\frac{1}{2} \int_{0}^{s} G_{n}^{\prime}\left(\frac{u}{2}\right) d u\right) \\
=\frac{d}{d s} \frac{1}{2} \int_{0}^{s} \sum_{n=1}^{\infty} \mu^{n} G_{n}^{\prime}\left(\frac{u}{2}\right) d u=\frac{1}{2} \sum_{n=1}^{\infty} \mu^{n} G_{n}^{\prime}\left(\frac{s}{2}\right),
\end{gathered}
$$

and the second derivative is handled in the same way.

Remark 2.3: It might be quite interesting if the recursion formula of Takács [14] for $M_{n}\left(=n ! G_{n}(0)\right)$ could be derived directly from Theorem 2.4 .

Recapitulating, we have proved:

Theorem 2.7: With $G_{n}(s)$ as in Theorem 2.4, we have for $0 \leq n$,

$$
E\left(\left(\int_{0}^{y}\left|U_{y}(s)\right| d s\right)^{n} \mid \ell_{y}(0)=x\right)=n ! y^{\frac{3 n}{2}} G_{n}\left(x y^{-\frac{1}{2}}\right), 0 \leq x, 0 \leq y \leq 1
$$

where $U_{y}$ is a Brownian bridge of span $y$ and $\ell_{y}(0)$ is twice its local time at 0.

\section{Points of Contact with Perman and Wellner [9]}

As in [9], we set $A_{0}^{+}=\int{ }_{0}^{1} U^{+}(t) d t, A_{0}^{-}=\int{ }_{0}^{1} U^{-}(t) d t$, where $U$ is a standard Brownian bridge. Thus we have $\int_{0}^{1} U(t) d t=A_{0}^{+}-A_{0}^{-}$and $\int_{0}^{1}|U(t)| d t=A_{0}^{+}+$ $A_{0}^{-}:=A_{0}$, where the first is Gaussian with mean 0 , hence its law is easy to find, while the law of the second has been obtained through a series of papers - see $[9$, Introduction]. The moments $\mu_{k}:=E A_{0}^{k}$ follow from a recursion formula of L. Shepp (see [9, Theorem 5.1]), while $\mu_{k}^{+}:=E\left(A_{0}^{+}\right)^{k}$ follow from a recursion formula of Perman and Wellner [9, Corollary 5.1]. By combining the above results, one can derive the joint moments $\mu_{m, n}:=E\left(\left(A_{0}^{+}\right)^{m}\left(A_{0}^{-}\right)^{n}\right)$ for $m+n \leq 5$. For example, $\mu_{1,1}=\frac{1}{4}\left(\mu_{2}-E\left(A_{0}^{+}-A_{0}^{-}\right)^{2}\right)$. But for $m+n>5$ the method does not suffice because in the expansion of $E\left(A_{0}^{+} \pm A_{0}^{-}\right)^{6}$ there are already four distinct joint moments $\mu_{m, n}$ with $m+n=6$ (discounting 3 symmetries) while there are only three 'knowns' (including $\mu_{6}^{+}$).

All of these moments (including arbitrary $(m, n)$ in $\mu_{m, n}$ ) follow in principle by our method, by integrating out over the local time and bridge span variables $(x, y)$. But if we require explicit expressions not involving integrals (i.e., that the integrations involved be done explicitly) a surprising fact emerges: we can do the integrations precisely for the bridge moments treated already in [9] - neither more not less. And besides, our comprehensive recursion formula of Theorem 2.4 is doubtless more unwieldy to apply then the separate recursions noted above, which (apparently) do not follow easily from it. Thus our results, at present, seem to provide a strong

vindication of the results of [9] for the moment problems treated here.
The density of $\ell_{1}(0)$ (the local time of $|U|$ at 0 ) is given by $4^{-1} x \exp \left(-\frac{x^{2}}{8}\right)$, according to P. Lévy (see $\left[7\right.$, p. 236]), and so $\mu_{n}=n ! \int_{0}^{\infty} 4^{-1} x \exp \left(-\frac{x^{2}}{8}\right) G_{n}(x) d x$. This yields

Proposition 3.1: For $0<n$, we have with $d_{j, n}$ from Theorem 2.4 


$$
\mu_{n}=n ! \sqrt{\pi} \sum_{j=n}^{3 n-2} 2^{-1-\frac{j}{2}} \Gamma^{-1}\left(\frac{j+3}{2}\right) d_{j, n}
$$

Proof: Noting again that $\exp \left(-\frac{x^{2}}{4}\right)=D_{0}(x)=x^{-1} D_{1}(x)$, we have

$$
\begin{gathered}
\int_{0}^{\infty} 4^{-1} x \exp \left(-\frac{x^{2}}{16}\right) D_{-j}\left(\frac{x}{2}\right) d x=\int_{0}^{\infty} y D_{0}(y) D_{-j}(y) d y \\
=(1+j)^{-1} \int_{0}^{\infty}\left(D_{1} D_{-j}^{\prime \prime}-D_{-j} D_{1}^{\prime \prime}\right) d y \\
=(1+j)^{-1}\left(D_{1} D_{-j}^{\prime}-D_{-j} D_{1}^{\prime}\right]_{0}^{\infty}
\end{gathered}
$$

where we used $[15$, p. 350$]$ for the second equality. Now by substituting for the two derivatives (see Table 2.1ff.) this becomes by (2.15)

$$
\begin{aligned}
=(1+j)^{-1}( & \left.-j D_{1} D_{-j-1}-D_{-j} D_{0}\right]_{0}^{\infty}=(1+j)^{-1} D_{-j}(0) \\
= & (1+j)^{-1} \sqrt{\pi} 2^{-\frac{j}{2}} \Gamma^{-1}\left(\frac{j+1}{2}\right),
\end{aligned}
$$

and substituting these values into Theorem 2.4 completes the proof.

Remark: Using the values of $d_{j, n}$ from Table 2.1 for $n \leq 4$, we get values $\mu_{1}=\frac{1}{4} \sqrt{\frac{\pi}{2}}, \mu_{2}=\frac{7}{60}, \mu_{3}=\frac{21}{512} \sqrt{\frac{\pi}{2}}$ and $\mu_{4}=\frac{19}{720}$, in agreement with Table 1 of [9] (which continues to $n=10$ ).

To handle the moments $\mu_{+}^{k}$ (or $\mu_{k}^{-}$analogously) we note from [10] as in (1.0), that the $P^{\alpha, y}$-law of $X$ is the same as that of the (spatial) local time process of $|B|$ at the time when it reaches $\alpha$ at 0 , conditional on that time being $y$. Suppose that in this statement we replace $|B|$ by the reflected Brownian motion obtained from $B$ by excising the negative excursion, i.e., $B(x(t))$ where $\int_{0}^{x(t)} I_{(0, \infty)}(B(u)) d u=t$. Since this has the same law as $|B|$, it also has the same local time process (equality in law). But now the time when the local time at 0 reaches $\alpha$ is, in terms of $B$, the time spent positive at the instant $T(\alpha)$ when the local time of $B$ at 0 reaches $\alpha$. The local time of $B(x(t))$ at $x \geq 0$ at time $T(\alpha)$ is the same as that of $B$ (since the negative excursions do not contribute). Thus $P^{\alpha, y}$ is also the law of the local time of the process of positive excursions of $B$ (holding the value 0 during the negative excursions) at $T(\alpha)$ given that the time spent positive equals $y$ at the instant $T(\alpha)$. Since it is entirely defined from $B(x(t))$ is not hard to see that this remains true if the time spent negative at this instant, say $z$, is also given, in such a way that up to time $y+z, B$ is in law a Brownian bridge of span $y+z$ given that its local time at 0 is $\alpha$ and its time spent positive is $y$. But here $y+z$ may be fixed $(\geq y)$, and in particular we may set $y+z=1$. Thus we have shown the following (see also [10, Corollary 18]):

Lemma 3.2: The law $P^{\alpha, y}$ of (1.0) ff. is also the law of the local time process in parameter $x \geq 0$ of the standard Brownian bridge $U$ conditional on its local time at 0 being $\alpha$ and its time spent positive being $y$.

Next we need the law of time spent positive by $U$ conditional on local time $\alpha$ at 0 . This is easily recognized as the time spent positive by $B$ at time $T(\alpha)$ given $T(\alpha)=$ 1. As noted by $P$. Lévy, this is the law of $T_{1}\left(\frac{\alpha}{2}\right)$ given $T_{1}\left(\frac{\alpha}{2}\right)+T_{2}\left(\frac{\alpha}{2}\right)=1$, where 
$T_{i}\left(\frac{\alpha}{2}\right)$ are independent copies of $T\left(\frac{\alpha}{2}\right), i=1$ or 2 , and we have:

Lemma 3.3: (See also [7, Corollary 1]) The above law has density

$$
(4 \sqrt{2 \pi})^{-1} \alpha(y(1-y))^{-\frac{3}{2}} \exp \left(-\frac{\alpha^{2}\left(y-\frac{1}{2}\right)^{2}}{2 y(1-y)}\right), \quad 0<y<1
$$

Combining the two lemmas with Theorem 2.4 gives the following theorem.

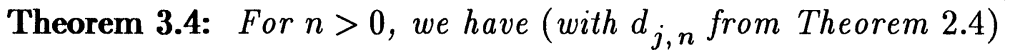

$$
\begin{aligned}
& \frac{\mu_{n}^{+}}{n !}= \\
& \quad \sqrt{\pi} \sqrt{2}-n \sum_{i=0}^{n-1} d_{n+2 i, n} \frac{2^{-i}}{(n+1-i)(n-1)}\left(\frac{2 n+i}{\Gamma\left(\frac{n+1}{2}+i\right)}-3 n \frac{\Gamma\left(\frac{3 n}{2}\right)}{\Gamma\left(\frac{3 n+1}{2}\right) \Gamma\left(\frac{n}{2}+i\right)}\right) .
\end{aligned}
$$

Remark: We are unable to simplify this further (see calculations below). Meanwhile we have checked it against [9, Table 1] for $n=1,2$ and 3 , where it gives $\mu_{1}^{+}=$ $\frac{1}{4} \sqrt{\frac{\pi}{2}}, \mu_{2}^{+}=\frac{1}{20}$, and $\mu_{3}^{+}=\frac{71}{4096} \sqrt{\frac{\pi}{2}}$, as required.

Proof: We have to integrate out $x$ and $y$ in the product

$$
x \exp \left(-\frac{x^{2}}{2}\right)(4 \sqrt{2 \pi})^{-1} x(y(1-y))^{-\frac{3}{2}} \exp \left(\frac{-x^{2}\left(y-\frac{1}{2}\right)^{2}}{2 y(1-y)}\right) y^{\frac{3 n}{2}} G_{n}\left(x y^{-\frac{1}{2}}\right)
$$

where $G_{n}(s)=\exp \left(\frac{s^{2}}{16}\right) \sum_{j=n}^{3 n-2} d_{j, n} D_{-j}\left(\frac{s}{2}\right)$. Fixing $j:=n+2 i, 0 \leq i \leq n-1$, and setting $(4 \sqrt{2 \pi})^{-1} d_{j, n}$ aside until the end, the $i^{\text {th }}$ term becomes

$$
\begin{aligned}
& \int_{0}^{\infty} x^{2} \int_{0}^{1} y^{\frac{3}{2}(n-1)}(1-y)^{-\frac{3}{2}}\left(\exp -\frac{x^{2}}{8 y(1-y)}\right)\left(\exp \frac{x^{2}}{16 y} D_{-(n+2 i)}\left(\frac{x}{2 \sqrt{y}}\right) d y d x\right. \\
& =8 \int_{0}^{\infty} z^{2}\left(\exp \frac{z^{2}}{4}\right) D_{-(n+2 i)}(z)\left(\int_{0}^{1} y^{\frac{3}{2} n}(1-y)^{-\frac{3}{2}} \exp -\frac{z^{2}}{2(1-y)} d y\right) d z \\
& =8 \int_{0}^{\infty} z^{2}\left(\exp \frac{z^{2}}{4}\right) D_{-(n+2 i)}(z)\left(\int_{0}^{\infty} v^{\frac{3 n}{2}}(1+v)^{-\frac{3 n+1}{2}}\left(\exp -\frac{z^{2} v}{2}\right) d v\right) d z
\end{aligned}
$$

where we used $z=\frac{x}{\sqrt{y}}$, then $v=\frac{y}{1-y}$. Here the inner integral is a Laplace transform in $\frac{z^{2}}{2}$, and it is found in [12, p. 13, \#9]. The result is that (3.2) equals

$$
\sqrt{2}^{3 n+12} \Gamma\left(\frac{3 n}{2}+1\right) \int_{0}^{\infty} z D_{-3 n-1}(z) D_{-n-2 i}(z) d z .
$$

Next $\quad$ we $\quad$ use $\quad z D_{-3 n-1}(z)=D_{-3 n}(z)-(3 n+1) D_{-3 n-2}(z)$ to write $(3.2)$ $=T_{1}+T_{2}$, where $T_{1}$ has the form

$T_{1}=c_{1} \int_{0}^{\infty} D_{-n-2 i}(z) D_{-3 n}(z) d z$ 


$$
\begin{aligned}
& =\frac{c_{1}}{2(n-i)} \int_{0}^{\infty} D_{-n-2 i} D_{-3 n}^{\prime \prime}-D_{-3 n} D_{-n-2 i}^{\prime \prime} d z \\
& =\frac{c_{1}}{2(n-i)}\left(-3 n D_{-n-2 i} D_{-3 n-1}+\left.(n+2 i) D_{-n-2 i-1} D_{-3 n}\right|_{0} ^{\infty}\right. \\
& =\frac{c_{1} \pi}{(n-i)} 2^{-2 n-i-\frac{1}{2}}\left(\Gamma^{-1}\left(\frac{3 n}{2}\right) \Gamma^{-1}\left(\frac{n+2 i+1}{2}\right)-\Gamma^{-1}\left(\frac{3 n+1}{2}\right) \Gamma^{-1}\left(\frac{n+2 i}{2}\right)\right),
\end{aligned}
$$

where we proceeded much as in Proposition 3.1 above. Similarly (replacing $D_{-3 n}$ by $D_{-3 n-2}$ ) we obtain

$$
\begin{gathered}
T_{2}=-c_{1}(3 n+1) \int_{0}^{\infty} D_{-n-2 i}(z) D_{-3 n-2}(z) d z \\
=\frac{-c_{1} \pi(3 n+1)}{(n-i+1)} 2^{-2 n-i-\frac{1}{2}\left((3 n)^{-1} \Gamma^{-1}\left(\frac{3 n}{2}\right) \Gamma^{-1}\left(\frac{n+2 i+1}{2}\right)\right.} \\
\left.\quad-(3 n+1)^{-1} \Gamma^{-1}\left(\frac{3 n+1}{2}\right) \Gamma^{-1}\left(\frac{n+2 i}{2}\right)\right) \\
=\frac{-c_{1} \pi}{n-i+1} 2^{-2 n-i-\frac{1}{2}\left(\Gamma^{-1}\left(\frac{3 n}{2}\right) \Gamma^{-1}\left(\frac{n+2 i+1}{2}\right)\right.} \\
\left.-\Gamma^{1}\left(\frac{3 n+1}{2}\right) \Gamma^{-1}\left(\frac{n+2 i}{2}\right)+\frac{1}{3 n} \Gamma^{-1}\left(\frac{3 n}{2}\right) \Gamma^{-1}\left(\frac{n+2 i+1}{2}\right)\right)
\end{gathered}
$$

Combining with $c_{1}=\sqrt{2}^{3 n+12} \Gamma\left(\frac{3 n}{2}+1\right)$, we obtain finally

$$
\begin{aligned}
& T_{1}+T_{2} \\
& \quad=\frac{\pi \sqrt{2}^{-n-2 i+11}}{(n-i+1)(n-i)}\left[\left(n+\frac{i}{2}\right) \Gamma^{-1}\left(\frac{n+2 i+1}{2}\right)-\Gamma\left(\frac{3 n}{2}+1\right) \Gamma^{-1}\left(\frac{3 n+1}{2}\right) \Gamma^{-1}\left(\frac{n+2 i}{2}\right)\right]
\end{aligned}
$$

(where the second term comes from combining the second terms of $T_{1}$ and $T_{2}$ ), and multiplication by $(4 \sqrt{2 \pi})^{-1} d_{j, n}$ yields the assertion.

Finally, we consider the mixed moments $\mu_{m, n}$. In addition to Lemmas 3.2 and 3.3 this requires:

Lemma 3.5: Given that the local time at 0 of a Brownian bridge $U$ is $\alpha$ and that the time spent positive is $y$, the conditional local time processes of $U$ in parameters $x \geq 0$ and $x \leq 0$ are independent.

Proof: The easiest way to see this is to use the well-known fact that the two corresponding local time processes of $B$ at time $T(\alpha)$ are independent (but also see [10, Corollary 18]). As in Lemma 3.2, conditioning their integrals to equal $y$ and $1-y$ respectively gives the joint law of the conditional local time processes of $U$, and it obviously preserves the independence.

From this lemma we see that the conditional local time process of $U$ has law $P^{\alpha, y} P^{\alpha, 1-y}$, where $P^{\alpha, y}$ governs the local time with parameter $x \geq 0$ and $P^{\alpha, 1-y}$ that with parameter $-x, x \geq 0$. In this way, one obtains:

Theorem 3.6: For $m>0, n>0$, we have (with $d_{j, n}$ from Theorem 2.4) $\frac{\mu_{m, n}}{m ! n !}=$ $\sum_{i_{1}=0}^{m-1} \sum_{i_{2}=0}^{n-1} d_{m+2 i_{1}, m^{d}+2 i_{2}, n} I\left(i_{1}, i_{2}\right)$, where 


$$
\begin{aligned}
I\left(i_{1}, i_{2}\right)=2 \sqrt{\frac{2}{\pi}} \int_{0}^{\frac{\pi}{2}}(\cos \theta)^{3 m+1}(\sin \theta)^{3 n+1} \\
\quad \times\left(\int_{0}^{\infty} D_{-m-2 i_{1}}(r \cos \theta) D_{-n-2 i_{2}}(r \sin \theta) e^{-\frac{r^{2}}{4}} r^{2} d r\right) d \theta .
\end{aligned}
$$

Note: Of course, for $m=0$ or $n=0$ this is subsumed by Theorem 3.4.

Proof: We need only multiply $(3.1)$ by $(1-y)^{\frac{3 m}{2}} G_{m}\left(x(1-y)^{-\frac{1}{2}}\right)$. The exponentials combine in a factor $\exp -x^{2}(16 y(1-y))^{-1}$, and routine changes of variables (set $x=2 \sqrt{y} z=2 \sqrt{1-y} v$ ) reduce the double integral to the expression of $I\left(i_{1}, i_{2}\right)$ in Cartesian coordinates $(z, v)$. The polar coordinate form given here seems slightly simpler.

While we do not know how to integrate $I\left(i_{1}, i_{2}\right)$ in general, we can reduce it for the case $m=n=1$ by use of Remark 2.1 to the effect that $G_{1}(s)=s^{-\frac{1}{2}} \exp \left(\frac{s^{2}}{8}\right) \operatorname{erfc}$ $\left(s 8^{-\frac{1}{2}}\right)$. Since the integration is, even there, a bit unorthodox, we shall conclude by presenting it. In this case, the combined exponent of exp reduces to 0 in the analog of (3.1) with $n=1$ and an extra factor $(1-y)^{\frac{3}{2}} G_{1}\left(x(1-y)^{-\frac{1}{2}}\right.$ ) (using the erfc representation of $\left.G_{1}\right)$, and we are left with altogether

$$
\begin{aligned}
\mu_{1,1} & =\frac{1}{16} \sqrt{\frac{2}{\pi}} \int_{0}^{\infty} x^{2}\left(\int_{0}^{1}\left(\operatorname{erfc} x(8 y)^{-\frac{1}{2}}\right)\left(\operatorname{erfc} x(8(1-y))^{-\frac{1}{2}}\right) d y\right) d x \\
& =\frac{1}{16} \sqrt{\frac{2}{\pi}} \int_{0}^{\infty} \int_{0}^{\infty} \exp \left(-t_{1}^{2}-t_{2}^{2}\right)\left(\int_{D\left(t_{1}, t_{2}\right)} x^{2} d x d y\right) d t_{1} d t_{2}
\end{aligned}
$$

where $D\left(t_{1}, t_{2}\right):=\left\{x(8 y)^{-\frac{1}{2}}<t_{1}, x(8(1-y))^{-\frac{1}{2}}<t_{2}\right\} \cap E$. By simple algebra we have $D\left(t_{1}, t_{2}\right)=\left\{x^{2}\left(8 t_{1}^{2}\right)^{-1}<y<1-x^{2}\left(8 t_{2}\right)^{-1}\right\} \cap E$, so the $y$-section of $D$ at $x$ is void unless $\frac{x^{2}}{8}\left(t_{1}^{-2}+t_{2}^{-2}\right) \leq 1$, in which case it has length $1-\frac{x^{2}}{8}\left(t_{1}^{-2}+t_{2}^{-2}\right)$. The integral becomes

$$
\frac{1}{16} \sqrt{\frac{2}{\pi}} \int_{0}^{\infty} \int_{0}^{\infty} \exp \left(-t_{1}^{2}-t_{2}^{2}\right) \int_{0}^{L} x^{2}-\frac{x^{4}}{8}\left(t_{1}^{-2}+t_{2}^{-2}\right) d x d t_{1} d t_{2}
$$

where $L=\sqrt{8} t_{1} t_{2}\left(t_{1}^{2}+t_{2}^{2}\right)^{-\frac{1}{2}}$, and integration over $x$ gives

$$
\frac{1}{16} \sqrt{\frac{2}{\pi}} \int_{0}^{\infty} \int_{0}^{\infty} \exp \left(-t_{1}^{2}-t_{2}^{2}\right)\left(\frac{1}{3}-\frac{1}{5}\right) L^{3} d t_{1} d t_{2}
$$


Expressing this in polar coordinates, it equals $\frac{4}{15 \sqrt{\pi}}\left(\int_{0}^{\infty} e^{-r^{2}} r^{4} d r\right) \int_{0}^{\frac{\pi}{2}} \cos ^{3} \theta \sin ^{3} \theta d \theta$, and by routine calculus it yields $\mu_{1,1}=(120)^{-1}$ as expected.

\section{Acknowledgement}

The author is very grateful to two anonymous referees for their helpful comments.

\section{References}

[1] Courant, R. and Hilbert, D., Methods of Mathematical Physics, Vol. 1, Interscience, New York 1953.

[2] Darling, D.A. and Siegert, A.J., The first passage problem for a continuous Markov process, Ann. Math. Stat. 24 (1953), 624-639.

[3] Feller, W., An Introduction to Probability Theory and Its Applications, Vol. 1, John Wiley and Sons, New York 1968.

[4] Itô, K. and McKean, H.P., Diffusion Processes and their Sample Paths, Academic Press, Inc., New York 1965.

[5] Kac, M., On the average of a certain Wiener functional and a related limit theorem in calculus of probability, TAMS 59 (1946), 401-414.

[6] Knight, F., Essentials of Brownian Motion and Diffusion, Math Surveys 18, Amer. Math. Soc., Providence, RI 1981.

[7] Knight, F.B., Inverse local times, positive sojourns, and maxima for Brownian motion, Astérisque 157-158 (1988), 233-247.

[8] Leuridan, C., Le théorème de Ray-Knight à temps fixe., Sem. de Prob. XXXII (1998), 376-396.

[9] Perman, M. and Wellner, J.A., On the distribution of Brownian areas, Ann. Appl. Probab. 6 (1996), 1091-1111.

[10] Pitman, J., The SDE solved by local times of a Brownian excursion or bridge derived from the height profile of a random tree or forest, The Annals of Probab. 27:1 (1999), 261-263.

[11] Revuz, D. and Yor, M., Continuous Martingales and Brownian Motion, Springer-Verlag, Berlin 1991.

[12] Roberts, G.E. and Kaufman, H., Table of Laplace Transforms, W.B. Saunders 1966.

[13] Rogers, C. and Williams, D., Diffusions, Markov Processes, and Martingales, Vol. 2, John Wiley and Sons, New York 1987.

[14] Takács, L., Random walk processes and their applications in order statistics, Ann. Appl. Probab. 2 (1992), 435-459.

[15] Whittaker, E.T. and Watson, G.N., A Course in Modern Analysis, Cambridge, London 1927. 


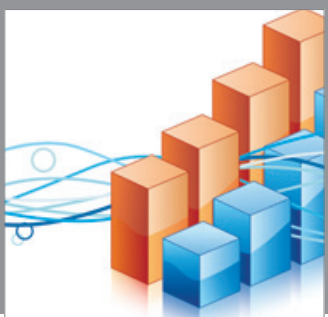

Advances in

Operations Research

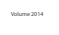

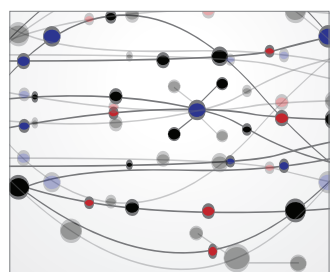

\section{The Scientific} World Journal
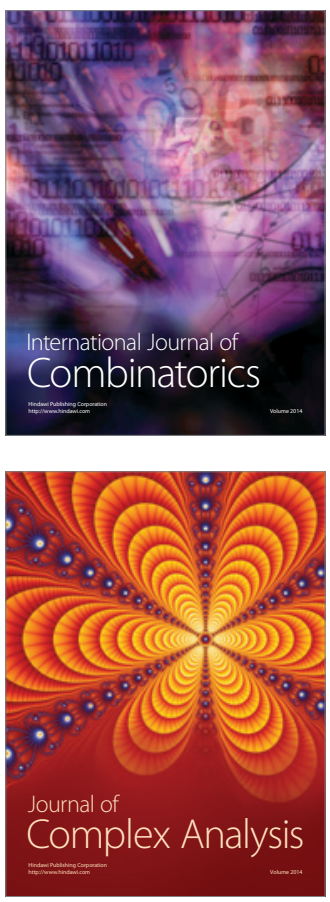

International Journal of

Mathematics and

Mathematical

Sciences
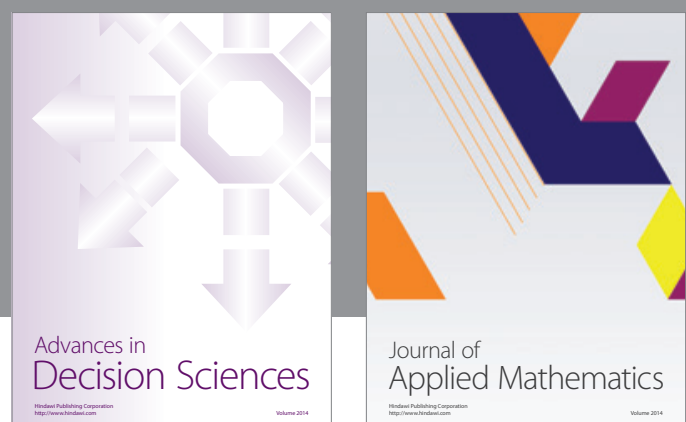

Journal of

Applied Mathematics
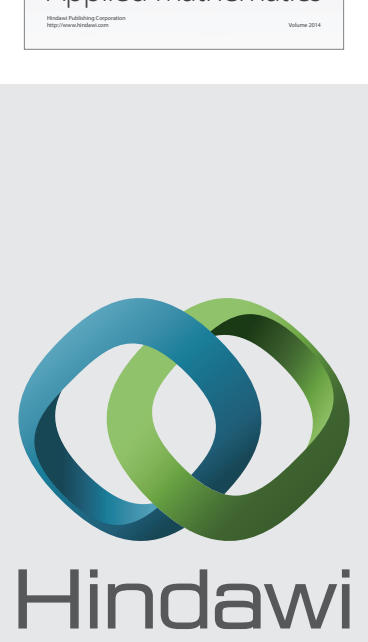

Submit your manuscripts at http://www.hindawi.com
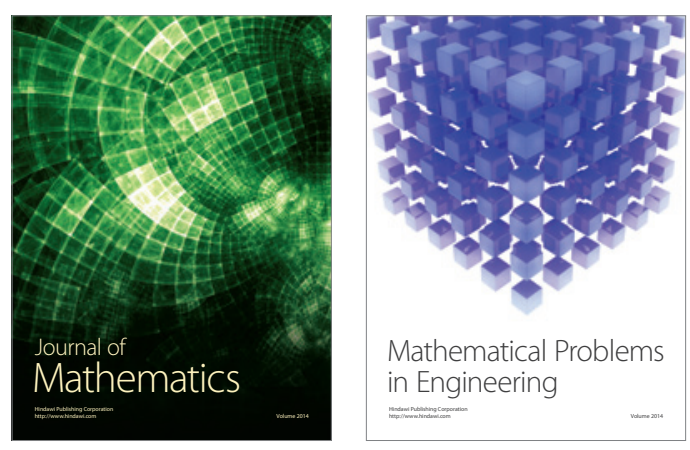

Mathematical Problems in Engineering
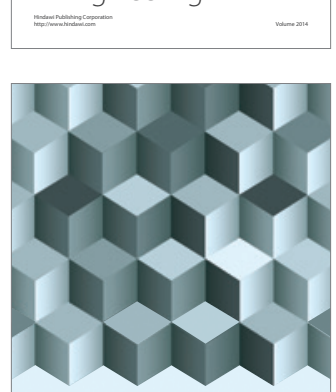

Journal of

Function Spaces
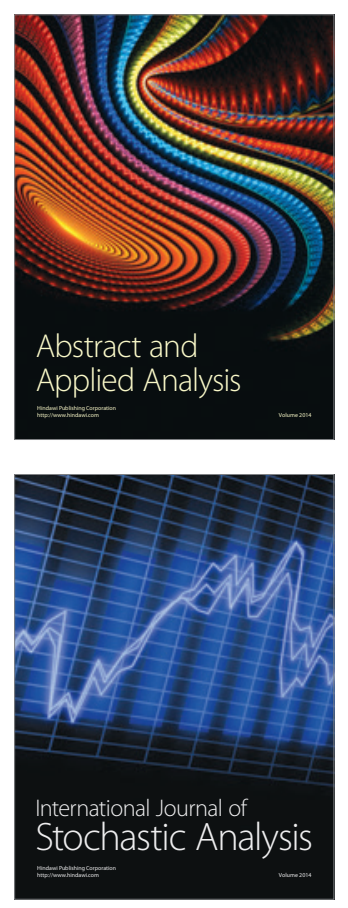

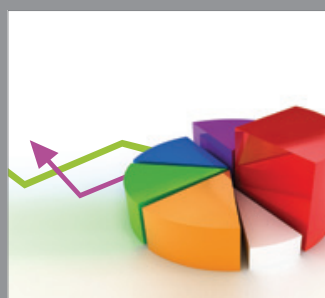

ournal of

Probability and Statistics

Promensencen
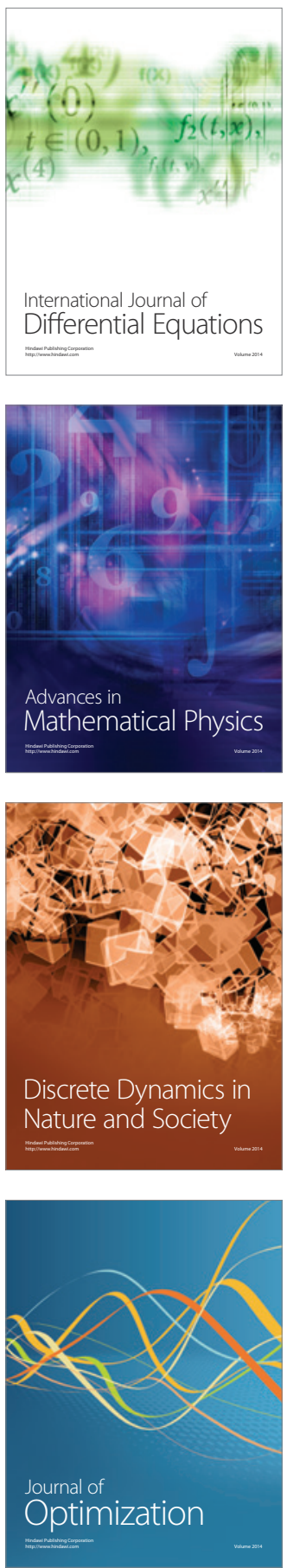